\title{
48th European Mathematical Genetics Meeting (EMGM) 2020
}

Lausanne, Switzerland, April 16-17, 2020

\section{Abstracts}

Guest Editors

Zoltan Kutalik, Lausanne

Matthew Robinson, Lausanne 


\section{A Simulation Study to Evaluate Existing Pleiotropy Detection Methods}

Adesoji $\mathrm{O}^{1}$, Nothnagel $\mathrm{M}^{2}$

${ }^{1}$ Cologne Center for Genomics, University of Cologne, Department of Statistical Genetics and Bioinformatics, Cologne, Germany; ${ }^{2}$ University Hospital Cologne, Cologne, Germany

Correspondence to: oadesoji@uni-koeln.de

Pleiotropy denotes the association of a hereditary unit (SNP, locus or gene) to multiple features or phenotypes. Detection of true biological pleiotropic locus, instead of mediated or spurious ones, may inform on the etiology of complex diseases as well as on their categorization. While many methods have been proposed in literature for the detection of pleiotropic locus, their applicability is to a large extent defined by the type of data available and their relative performance is yet unclear. Here, we investigated different forms of meta-analysis based approaches, namely classical metaanalysis (MA), conditional false discovery rate (cFDR; Andreassen et al., 2013; Liley \&Wallace, 2015), subset-based meta-analysis (ASSET; Bhattacharjee et al., 2012), and CPBayes (CPB; Majumdar et al.,2018). To this end, we performed a simulation study where we repeatedly simulated case-control data sets of varying sizes and with different underlying etiologies from a resampled population of 50,000 individuals of European ancestry based on the $1000 \mathrm{Ge}-$ nomes project. We considered various combinations of factors, including varying effect sizes, variant allele frequencies, numbers of disease SNPs, varying sample sizes and the degree of genetic overlap between the phenotypes, and assessed their impact on power and type I error of these methods. While work is ongoing, we will present preliminary results from our simulation study.

\section{Matrix Factorisation Reveals Cell-Specific Trans-Acting Regulatory Variants Controlling Modules of Co-Expressed Genes}

Kolberg L, Alasoo K

University of Tartu, Tartu, Estonia

Correspondence to: kaur.alasoo@ut.ee

A major challenge in human genetics is translating non-coding GWAS loci to mechanistic understanding about the disease causing processes. Local gene expression quantitative trait loci (ciseQTLs) regularly implicate multiple putative target genes whose disease relevance and function is often poorly understood. In contrast, genetic variants that are associated with the expression of multiple target genes in trans, have the potential to directly identify the cellular processes affected by disease variants. However, trans-eQTLs are difficult to detect due to small sample sizes of current eQTL datasets and large number genes tested. Here, we have jointly analysed five eQTL dataset profiling gene expression in naive and stimulated B-cells, T-cells, monocytes, neutrophils and platelets from up to 710 individuals. To improve interpretability of trans-eQTLs and reduce multiple testing burden, we used five matrix factorisation techniques to infer gene co-expression modules from expression data. We find that trans-eQTLs regulating co-expression modules are highly cell type specific and are often detected by a single matrix factorisation approach. These include established trans-eQTLs, such as the platelet-specific $A R H$ GEF3 locus associated with mean platelet volume and monocyte-specific IFNB1 locus associated with activation of genes downstream of the type 1 interferon signalling pathway upon LPS stimulation. Co-expression modules under cell-type specific genetic control also exhibit higher variance in the cell types where the associations are detected, suggesting that our results are not simply an artefact of limited power. Thus the contexts in which transeQTL are active are likely to be missed when studying bulk tissues such as whole blood.

\section{eQTL Catalogue: A Compendium of Uniformly Processed Human Gene Expression and Splicing QTLs}

\section{Kerimov N, Alasoo K}

University of Tartu, Tartu, Estonia

Correspondence to: kaur.alasoo@ut.ee

An increasing number of gene expression quantitative trait locus (QTL) studies have made summary statistics publicly available, which can be used to gain insight into human complex traits by downstream analyses such as fine-mapping and colocalisation. However, differences between these datasets in their variants tested, allele codings, and in the transcriptional features quantified are a barrier to their widespread use. Here, we present the eQTL Catalogue, a resource which contains quality controlled, uniformly re-computed QTLs from 19 eQTL publications. In addition to gene expression QTLs, we have also identified QTLs at the level of exon expression, transcript usage, and promoter, splice junction and 3 ' end usage. Our summary statistics can be downloaded by FTP or accessed via a REST API and are also accessible via the Open Targets Genetics Portal. We demonstrate how the eQTL Catalogue and GWAS Catalog APIs can be used to perform colocalisation analysis between GWAS and QTL results without downloading and reformatting summary statistics. New datasets will continuously be added to the eQTL Catalogue, enabling karger@karger.com

www.karger.com/hhe

Karger"
C 2020 S. Karger AG, Basel

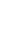


systematic interpretation of human GWAS associations across a large number of cell types and tissues. The eQTL Catalogue is available at https://www.ebi.ac.uk/eqtl/.

\section{Efficiency of Statistical Models to Favor the Selection of Important Specific Characters}

Almorza $D^{3}$, Kandus $M^{1}$, Prada Oliveira $A^{3}$, Salerno $\mathcal{J}^{2}$

${ }^{1}$ INTA, Buenos Aires, Argentina; ${ }^{2}$ University del Salvador, Buenos Aires, Argentina; ${ }^{3}$ University of Cádiz, Cádiz, Spain

Correspondence to: david.almorza@uca.es

To determine the most efficient selection of specific characters such as the production of grains in maize, it is important to know the gene effects that regulate it. The objective of this work was to apply two models to evaluate combinatorial aptitude (CA) in grain production: Genotype main effects and Genotype by Environment interaction model (GGE) adapted by Yan and Hunt (2002), and linear model proposed by Griffing (1956) adapted to the analysis of the CA. The dialectic crossings were performed, between eight lines and all possible combinations were evaluated (twenty eight obtained hybrids and their progenitor lines). The trials were conducted two consecutive years in one location using a randomized complete block design. The GGE model showed that the general combinatorial aptitude (GCA) effects of the lines differed from year to year, while the specific combinatorial aptitude(ECA) effects of hybrids showed more coincidences between the two years. The Griffing model highlighted significant effects of ECA for the study variable in both years. The high genotypexenvironment interaction presented by grain production would explain these results. A partial coincidence between both models was observed in the GCA and ECA for the first year and was significant for the second year. In the GGE biplot model, the first two principal components explained more than $50 \%$ in the variation for both years. These results confirm previous work that demonstrates the usefulness of the GGE model in the evaluation of CA, further confirming that the multivariate analysis has greater advantages compared to linear models.

\section{A Flexible and Shared Information Bayesian Joint Fine-Mapping Approach for Multiple Quantitative Traits}

Hernández $N^{2}$, Newcombe $P^{2}$, Sandhu $M^{1}$, Wallace $C^{3}$, Asimit $\mathcal{J}^{2}$

${ }^{1}$ Department of Medicine, University of Cambridge, Cambridge, UK; ${ }^{2} \mathrm{MRC}$ Biostatistics Unit, University of Cambridge, Cambridge, UK; ${ }^{3}$ Department of Medicine, University of Cambridge, Cambridge, UK

Correspondence to: jennifer.asimit@mrc-bsu.cam.ac.uk

Hundreds of genetic variants have been identified as associated with a spectrum of diseases and related traits, but pinpointing likely causal variants (fine-mapping) has been complicated by extended linkage disequilibrium (LD) between genetic variants, as well as finite sample sizes. Fine-mapping inaccuracies often occur when there are two or more distinct causal variants that are both correlated with a single non-causal variant. This motivates the development of joint fine-mapping that leverages information between multiple traits with shared aetiology in a Bayesian framework. Prior model probabilities for each trait can be formulated to favour combinations of models which share causal variants to enable borrowing information between outcomes. Using summary statistics, flashfm (FLexible And SHared information FineMapping) fine-maps association signals for multiple quantitative traits, allowing for missing trait measurements and related individuals. Our method does not assume that the traits must share any or all causal variants. Simulation studies demonstrate that this joint approach has greater accuracy than single outcome analyses when shared causal variants are present, yet no precision loss if there is no sharing. We jointly fine-map association signals for 34 cardiometabolic traits in a Uganda cohort that includes related individuals. Our proposed approach is computationally efficient and exploits relatedness of traits in current sample sizes to increase fine-mapping resolution, at lower cost and more feasible, than collecting larger samples.

\section{Assessment of a Potential Mediation Effect of FMD on the Genetic Association with SCAD}

\section{Berrandou T, Bouatia-Naji N}

Inserm, Paris, France

Correspondence to: takiy.berrandou@inserm.fr

Spontaneous coronary artery dissection (SCAD) is an underdiagnosed coronary syndrome. Fibromuscular dysplasia (FMD) is a non-atherosclerotic vascular stenosis that results in hypertension or stroke. Several clinical features are shared by these diseases including higher prevalence among women. We estimate that asymptomatic FMD is reported in arterial beds of $50 \%$ of SCAD patients. However, there is little, if any, SCAD events in FMD according to the existing series of patients, suggesting SCAD as a potential complication of FMD in the coronary bed. We have recently identified a common genetic risk locus, rs9349379 in PHAC$T R 1$, that significantly associate with risks for FMD $\left(\beta_{\mathrm{add}}=0.33\right.$ $95 \% \mathrm{CI}=0.22-0.43)$ and SCAD $\left(\beta_{\mathrm{add}}=0.5195 \% \mathrm{CI}=0.40-0.62\right)$. Here we aimed to assess the potential mediation role of FMD in PHACTR1 association with SCAD. We analyzed data from two studies: SCAD (292 cases, 3468 controls) and FMD (646 cases, 1487 controls). We used logistic regression models (log-additive) adjusted for sex and bootstrapping algorithm implemented in the R package "mma". We estimate a total effect of PHACTR 1 on SCAD risk of 0.46 (95\% CI $=0.27-0.67)$, a direct effect of $0.39(95 \% \mathrm{CI}=0.21-0.62)$ and an indirect effect (through FMD) of 0.07 (95\%CI=0.05-0.10). The proportion of genetic association mediated through FMD is 0.15 (95\%CI $=0.10-0.27)$. Our results indicate that PHACTR1 influences more strongly the genetic risk for SCAD directly than through an indirect effect due to the presence of FMD supporting true pleiotropy. Further analyses are ongoing to assess how other shared genetic loci and suspected exposure mediators (e.g, hormones fluctuation) influence the genetic risk for SCAD. 


\section{Large-Scale Identification of Links between the Human Blood Metabolome and Transcriptome}

\author{
Beuchel C, Kirsten H, Tönjes A, Löffler M, Thiery J, \\ Ceglarek U, Scholz M \\ University of Leipzig, Leipzig, Germany \\ Correspondence to: cfbeuchel@imise.uni-leipzig.de
}

Investigating the interaction of the human blood transcriptome and metabolome is of high relevance to understand molecular factors of metabolism-associated diseases such as obesity and type 2 diabetes.

We here integrate blood metabolome and transcriptome data of three large cohorts (LIFE Heart, LIFE Adult and the Sorb study). Metabolome data were measured by LC-MS/MS in dried whole blood or plasma. Transcriptome data were measured by Illumina HT12v4 gene-expression arrays in whole blood or PBMC. Data were harmonized and pre-processed using pipelines proposed by us to account for relevant features of the data and to identify potential confounders of the associations between these omics layers. We searched for associations between 97 metabolomics features (single metabolites and derived quotients) and 26,042 high-quality transcripts in a total of 7,706 subjects.

Meta-analysis revealed a total of 37,461 associations involving 8,579 genes and 72 metabolites. Most associations were observed for acetylcarnitine (2,187 significant genes). Conversely, on gene level, the zinc-finger protein coding gene BCL11A was associated with 56 metabolites.

We analysed mediation effects of the 61,731 gene-metabolite pairs on BMI and T2D by bi-directional mediation analysis. Exemplary, associations between the amino acids Sarcosine and Leucine/Isoleucine and BMI and T2D are strongly mediated by CCAR1, a key coregulatory of adipocyte differentiation. Pathway analysis of strongly T2D-linked metabolite-gene pairs revealed enrichment of genes involved in numerous immune system pathways.

By our analyses, we propose an atlas of possible molecular interactions and drivers of obesity and T2DM to be verified in pre-clinical models or interventional studies.

\section{Finding the Best Testing Units in Rare Variant Association Tests: An Approach Using Variant Pathogenicity Scores}

\author{
$\underline{B o c h e r}^{4}$, Ludwig $T^{1}$, Tournier-Lasserve $E^{3}$, Marenne $G^{2}$, Génin $E^{2}$ \\ ${ }^{1}$ UMR1078, CHU Brest, Brest, France; '2UMR1078, CHU Brest, \\ Brest, France; ${ }^{3}$ UMR囚S1161, CHU Brest, Brest, France; ${ }^{4}$ UMR1078, \\ Université de Bretagne Occidentalem Brest, Brest, France \\ Correspondence to: ozvan.bocher@univ-brest.fr
}

Different methods have been proposed to test for association between disease and rare variants when sequence data are available on cases and controls. These methods require first the definition of the testing units and then the selection of eligible variants into these testing units. When focus is on the exonic parts of the genome, typical testing units are the different genes delimited by their beginning and ending positions on the genome. However, this is not always the most optimal way of defining testing units as there might be some different parts within a gene with different functionality. As an alternative, sliding window approaches have been developed that do not require to pre-select the testing units based on functional annotations. These methods however are computationally intensive with results that could differ depending on the choice of window sizes. Here, we propose to group rare variants into genomic units using pathogenicity scores of variants observed in GnomAD. We show on an exome-wide analysis of Moyamoya disease that using these regions enables to find more significant regions than using the gene definition by pinpointing the specific region of the gene with the highest association signal. In this example, our method outperformed WGScan, a recently proposed sliding window approach. These encouraging results obtained on the coding regions of the genome suggest that a similar approach could also be used in the non-coding regions of the genome where we dramatically lack functional annotations to choose rare variant testing units.

\section{Closed Form Haplotype Frequency Estimation with Applications to the KIR Loci \\ van der Burg $L^{2}$, Baldauf $H^{1}$, Schetelig $J^{1}$, de Wreede $L^{2}$, Böhringer $S^{2}$ \\ ${ }^{1}$ DKMS, Tübingen, Germany; ${ }^{2}$ Leiden University Medical Center, Leiden, The Netherlands \\ Correspondence to: s.boehringer@lumc.nl}

Haplotype analysis can complement SNP based analyses, especially in genetically complex regions such as immune loci (KIR). However, such analyses are hampered by the computational burden, including the exponential growth in the number of possible haplotypes with the number of loci and slow convergence of expectation-maximization (EM) algorithms that are popular for problems with missing information, here phase information. We address these problems by developing a closed form formula for haplotype frequency estimation and by developing efficient grouping strategies that limit the number of haplotypes.

The closed form formula is developed by solving the problem for the bi-allelic, bi-locus setting where haplotype frequencies can be related to the sample covariance between SNPs and allele frequencies. The solution is then generalized to any number of loci and alleles. We prove consistency of the estimator and show in simulations that the estimator is fully efficient. The presented algorithm allows for grouping of haplotypes either in a data dependent way (e.g. haplotype frequencies), pre-specified classes (e.g. phylogenetic trees) or a combination thereof.

To illustrate the approach, we analyze a KIR (Killer-cell Immunoglobulin-like Receptor) data set measured on a cohort of healthy donors who donated hematopoietic cells for patients with acute myeloid leukemia or myelodysplastic syndromes. We demonstrate the effect of different grouping strategies to reduce the number of predictors in down-stream regression analyses. Comparing with a standard EM, we show a speedup of more than 100 for more than 6 loci.

Our algorithms allow to perform genome-wide haplotype analyses on a routine basis. 


\section{The Causal Relevance of Fatty Acids in the Development of Cardiovascular Diseases}

Borges $M^{2}$, Haycock $P^{2}$, Hingorani $A^{1}$, Lawlor $D^{2}$

${ }^{1}$ University College London, London, UK; ${ }^{2}$ University of Bristol, Bristol, UK

Correspondence to: m.c.borges@bristol.ac.uk

There is an ongoing debate on the role of fatty acids in the development of cardiovascular diseases (CVDs). We have used Mendelian randomization to probe the effect of fatty acids on CVDs risk [i.e.atrial fibrillation (AF), stroke, coronary artery disease (CAD), heart failure (HF); $\mathrm{N}=47,309-80,945$ cases/468, 141-930, 014 controls]. SNPs nearby genes coding for rate-limiting enzymes in fatty acids biosynthesis (e.g.FADS1/2, SCD, ELOVL2) were used as instruments due to their well-established role and impact on the fatty acids pool. Increased activity of FADS1/2 was related to modestly increased risk of $\mathrm{CAD}(\mathrm{OR}=1.03$ per $\mathrm{SD}$ increase in arachidonic acid;95\%CI:1.00-1.05), HF (OR=1.02; 95\%CI:1.00-1.04) and AS (OR=1.02;95\%CI:1.00-1.04), while increased activity of $S C D$ was related to decreased risk of CAD $(\mathrm{OR}=0.70$ per SD increase in palmitoleic acid; 95\%CI:0.58-0.85). There was no strong evidence that ELOVL2 activity was related to any of the CVD endpoints. To gain insights into potential mechanisms linking fatty acids biosynthesis to CVDs, we conducted a phenome-wide scan of the top SNP of each genomic region of interest $\left(\mathrm{P}<5^{\star} 10-8\right)$. SNPs were exclusively (i.e.ELOVL2) or almost exclusively (i.e.SCD) related to fatty acids traits, while FADS1/2 SNP was also related to numerous non-fatty acids traits [e.g. fasting glucose, blood lipids (HDLc, LDLc, TG), haematological traits, height and $\mathrm{BMD}]$, some of which could plausibly mediate the effects of FADS1/2 activity on CVDs. Our findings provide indirect evidence of the involvement of fatty acids on CVDs development and suggest enzymes coded by FADS1/2 and SCD as potential targets for primary prevention of CVDs.

\section{Localizing Components of Shared Transethnic Genetic Architecture of Complex Traits from GWAS Summary Data}

Burch $K^{2}$, Shi $H^{1}$, Johnson $R^{2}$, Freund $M^{2}$, Kichaev $G^{2}$, Mancuso $N^{3}$, Manuel $A^{2}$, Dong $N^{2}$, Pasaniuc $B^{2}$

${ }^{1}$ Harvard T.H. Chan School of Public Health, Boston, MA, USA;

${ }^{2}$ University of California, Los Angeles, CA, USA; ${ }^{3}$ University of

Southern California, Los Angeles, CA, USA

Correspondence to: kathrynburch@ucla.edu

Despite strong transethnic genetic correlations reported in the literature for many complex traits, the non-transferability of polygenic risk scores across populations implies that (1) LD tagging and/or allele frequencies of shared causal variants vary across populations; (2) a sizeable number of causal variants are population-specific; and/or (3) causal effect sizes vary across populations due to, for example, population-specific gene-environment interactions. We propose an approach that models GWAS summary data for one trait in two populations to estimate genome-wide proportions of population-specific/shared causal SNPs. In simulations across various genetic architectures, we show that our approach yields approximately unbiased estimates with in-sample LD and slight upward-bias with out-of-sample LD. We analyze 9 complex traits in individuals of East Asian and European ancestry, restricting to common SNPs (MAF $>5 \%$ ), and find that most common causal SNPs are shared by both populations. Using the genome-wide estimates as priors in an empirical Bayes framework, we perform fine-mapping and observe that high-posterior SNPs (for both the population-specific and shared causal configurations) have highly correlated marginal effects in East Asians and Europeans. In population-specific GWAS risk regions, we observe a 2.8x enrichment of shared high-posterior SNPs, suggesting that population-specific GWAS risk regions harbor shared causal SNPs that are undetected in the other GWAS due to differences in LD, allele frequencies, and/or sample size. Finally, we report enrichments of shared high-posterior SNPs in 53 tissue-specific functional categories and find evidence that SNP-heritability enrichments are driven largely by many low-effect common SNPs.

\section{Use of External Controls for Whole Genome Sequencing Data - Quality Control Considerations}

Dandine-Roulland $C^{1}$, Resch $L^{2}$, Sandron $F^{2}$, Sahbatou $M^{4}$, Bacq-Daian $D^{2}$, Blanché $H^{4}$, Meyer $V^{2}$, Deleuze J- $F^{3}$, Le Floch $E^{2}$

${ }^{1}$ Centre National de Recherche en Génomique Humaine, Institut de Biologie François Jacob, Direction de la Recherche Fondamentale, CEA, Paris, France; ${ }^{2}$ Centre National de Recherche en Génomique Humaine, Institut de Biologie François Jacob, Direction de la Recherche Fondamentale, CEA, Evry, France; ${ }^{3}$ Centre National de Recherche en Génomique Humaine, Institut de Biologie François Jacob, Direction de la Recherche Fondamentale, CEA, Evry, France / Centre d'Etude du Polymorphisme Humain, Fondation Jean Dausset, Paris, France / CREFIX, Evry, France; ${ }^{4}$ Centre d'Etude du Polymorphisme Humain, Fondation Jean Dausset, Paris, France

Correspondence to: dandine@cng.fr

Using external controls in Sequencing Association Studies, which avoids sequencing costs for controls, is more and more common. But, due to the fast technical development, case and external control datasets are generally not produced using the same technology. In this context, we tried to select controls for 49 French cases among 855 individuals from the same population (France GenRef). After applying classical statistical quality controls (QC) on variants - sequencing quality score (Q10), callrate, HardyWeinberg Equilibrium - we performed a Principal Component Analysis (PCA) and observed that cases and external controls are discriminated in two groups. Indeed, the two considered datasets differ for two main points; DNA sample type - blood and cell lines for controls and cases respectively - and Illumina sequencer used - HiSeq X and 2000 respectively. Using 9 controls sequenced on the two sequencers, we explored these two points. Firstly, PCA discrimination between the two sequencers stayed visible between the 9 duplicated individuals suggesting that DNA sample types do 
not explain completely our observations. Secondly, to explore the sequencer technological differences which can impact sequencing quality in some regions, we considered the influence on PCA of several additional bioinformatic QC measures - mappability, low quality variants identified by GNOMAD, segmental duplications, low complexity regions and VQSR (GATK). We observed that all variants excluded by these QCs (approximately two thirds of variants) explain the visible sequencer effect, in particular GNOMAD low quality variants. In conclusion, the combination of statistical and bioinformatic QCs seems necessary for external control use in sequencing association analysis.

\section{Simultaneous Estimation of Bi-Directional Causal Effects and Heritable Confounding from GWAS Summary Statistics}

\section{Darrous L, Mounier N, Kutalik Z}

University Center for Primary Care and Public Health, University of Lausanne, Lausanne, Switzerland

Correspondence to: liza.darrous@unil.ch

Introduction: Mendelian Randomisation (MR), an increasingly popular method that estimates the causal effects of risk factors on complex human traits, has seen several extensions that relax its basic assumptions. However, most of these extensions suffer from two major limitations; under-exploitation of genome-wide markers, and sensitivity to the presence of a heritable confounder of the exposure-outcome relationship. To overcome these limitations, we propose a Latent Heritable Confounder MR (LHC-MR) method applicable to association summary statistics, which simultaneously estimates bi-directional causal effects, direct heritability, and confounder effects while accounting for sample overlap.

Results: We demonstrate that LHC-MR outperforms several existing MR methods in terms of bias and variance for a wide range of simulation settings and apply it to summary statistics of $13 \mathrm{com}$ plex traits. Besides several concordant results, LHC-MR unravelled new mechanisms (how being diagnosed for certain diseases might lead to improved lifestyle) and revealed potential false positive findings of standard MR methods (apparent causal effect of body mass index on educational attainment may be driven by a strong ignored confounder). Phenome-wide search to identify LHC-implied heritable confounders showed remarkable agreement between the LHC-estimated causal effects of the latent confounder and those for the potentially identified ones. Finally, LHC-MR naturally decomposes genetic correlation to causal effect-driven and confounder-driven contributions, demonstrating that the genetic correlation between systolic blood pressure and diabetes is predominantly confounder-driven.

Conclusion: LHC-MR, a structural equation mixed effect model, is a novel method that tackles both causal inference with increased precision and the genetic architecture of complex disease.

\section{Circular Code and Genetic Code \\ Dila G ${ }^{2}$, Michel $C^{2}$, Thompson ${ }^{2}$, Poch $O^{2}$, Mayer $C^{1}$, Ripp $R^{2}$ \\ ${ }^{1}$ University of Paris Diderot, Paris, France; ${ }^{2}$ University of \\ Strasbourg, Strasbourg, France \\ Correspondence to: gopal.dila@etu.unistra.fr}

The circular code $X$ is an error-correcting code that has the ability to retrieve, maintain and synchronize the reading frame in genes (Arquès and Michel, 1996, Michel 2015, Michel et al., 2017). Motifs of the circular code $X$ are found to be significantly enriched in protein-coding genes of most organisms, from prokaryotes to eukaryotes. It has been suggested that these $X$ motifs may represent evolutionary remnants of a primordial code originally used for gene translation. In an analysis involving complete genomes from four mammals and nine yeast species, we demonstrated specific evolutionary pressures on $X$ motifs and highlighted important properties of $X$ motif conservation at the encoded amino acid level (Dila et al., Biosystems 2019). We observed a significant correlation between the number of $X$ motifs present and increased gene expression. In another large-scale study involving 133 organisms representing the three domains of life, we identified 32 universal $X$ motifs in ribosomal RNA (rRNA), most of which are located in regions involved in important ribosome functions, notably the peptidyl transferase center (PTC) and the decoding center (Dila et al., RNA 2019). Based on our results, we propose that circular codes represented an important step in the emergence of the modern genetic code, allowing simultaneous coding of amino acids as well as synchronization of the reading frame in primitive translation systems, prior to the advent of more sophisticated mechanisms. Our ongoing studies are now focused on the hypothesis that circular code motifs continue to act as functional elements within the coding regions of extant genomes.

\section{Integrating Three-Dimensional Organization of the Genome into Association Studies: Single SNP, SNP Set and SNP-SNP Interaction Tests}

\author{
Djebali $S^{2}$, Corvol J-C, Martinez $M^{2}$ \\ ${ }^{1}$ U1 127, Inserm, Paris, France; ${ }^{2}$ U1220, Inserm, Paris, France \\ Correspondence to: sarah.djebali@inserm.fr
}

In the context of complex genetic diseases, if genome-wide association studies (GWAS) have provided many important variants, GWAS unfortunately often fail in identifying variants that are rare or with very small effects. For this reason, there have been several attempts to group variants into functional units of the genome, most often genes, hoping to gain statistical power (SNP-set methods, PMID: 23741293). While these approaches have proven useful, we think it is important to go beyond genes and consider other functional units of the genome. Indeed many international consortia such as ENCODE, Epigenome Roadmap and FANTOM have revealed a plethora of regulatory elements such as enhancers, repressors and insulators, and shown that enhancers of a cell type related to a disease are almost always enriched in variants associated to this disease (PMID: 25693563). 
In 2018, Wu and Pan (PMID: 29728367) used two different SNP-set methods to group genes belonging to enhancers and to enhancer/gene relationships before GWAS on schizophrenia data. We wish to explore the same kind of approach on raw data from Parkinson's disease using regulatory relationships recently generated on four types of neural cells (PMID: 31367015). We also aim to extend this approach to identify non-independent genotype distributions in patients vs controls as well as within clusters of patients.

\section{Simulation Study of Multi-Predictor Epigenome- Wide Association Method Performance}

\section{Draisma H, Kaakinen M, Prokopenko I}

\section{University of Surrey, Guildford, UK}

Correspondence to: h.draisma@surrey.ac.uk

We developed the methylSCOPA software for fast multipredictor epigenome-wide association study (MP-EWAS), which uses multiple predictors of DNA methylation (DNAm) at a site in an ordinal regression model. Here, we evaluate the method's type I error rate (alpha) and power in simulation studies. For sample sizes of 1,000 and 5,000 individuals, we simulated DNAm data using the R package "quantroSim" which implements the Langmuir adsorption model. From a multivariate normal distribution we simulated data for two anticorrelated-to-correlated $(\mathrm{r}=-0.9 /-$ $0.5 /-0.3 /-0.1 / 0 / 0.1 / 0.3 / 0.5 / 0.9)$ continuous phenotypes having varying strength and direction of association $(-0.1 \leq$ regression coefficient $\leq 0.1$ ) with simulated DNAm data beta values. We estimated our method's alpha and power using the full model including both phenotypes by calculating the proportion of methylSCOPA analyses in which $P<0.05$ for the test of association. In addition, we compared the power for the full model with that of the single-predictor test that is included in methylSCOPA multi-predictor analysis, by calculating the proportion of tests for which the minimum $P$ value from the two analyses for each DNAm probe was smaller than 0.025 , i.e. the nominal $P$-value threshold of 0.05 Bonferroni-corrected for two tests performed. Consistent with the results of simulation studies for related multi-phenotype genome-wide association analysis methods, in the current simulation studies the methylSCOPA full model displayed markedly higher power over single-predictor analysis in particular when predictors were strongly correlated or anticorrelated. We designed and performed simulation studies to evaluate alpha and power of the methylSCOPA MP-EWAS method, demonstrating its enhanced power over single-predictor analysis. Funding: WT205915; H2020-SC1-2019-874739.

\section{Prediction and Interpretation of the Disease and Mortality Risks in Biobank Cohorts}

Fischer K, Mändul M, Läll K

University of Tartu, Tartu, Estonia

Correspondence to: Krista.Fischer@ut.ee

Polygenic Risk Scores (PRS) have shown the potential of improving existing risk-stratification algorithms and, for some cases could lead to more efficient screening programmes. However, to be able to estimate accurately the anticipated benefit of -omicsbased predictors in practice, more attention should be paid to the methodology of risk prediction. In this presentation, some methodological challenges will be highlighted with possible options to tackle them. The ideas will be illustrated using the examples of PRS-based predictions of the risk of Breast Cancer and Type 2 Diabetes in the Estonian Biobank.

When the outcome of interest is all-cause mortality, the definition of (cumulative) risk is straightforward, but in all other cases (disease incidence, cause-specific mortality) there are competing events that need to be taken into account. A common practice in PRS-related publications is to treat competing events as censoring, but this will lead to exaggerated estimates of the absolute risks and the effect of PRS. However, when competing risks are accounted for, the interpretation of the resulting estimates, for instance in genomic counselling situation, can be complicated.

To estimate the PRS effect, the Cox Proportional Hazards model is usually the most popular option. Whereas mostly valid for effect estimation, it does not provide closed form out-of-sample predictions for the survival probabilities. Using the Estonian Biobank data, we will compare a range of parametric options that can be considered when implementing the -omics-based risk predictions in personalized medicine.

\section{Population Stratification in Polygenic Risk Prediction Models for Coronary Artery Disease}

\author{
Gola $D^{3}$, Erdmann ${ }^{3}$, Müller-Myhsok $B^{1}$, Schunkert $H^{2}$, König ${ }^{3}$ \\ ${ }^{1}$ Max Planck Institute of Psychiatry, Munich, Germany; \\ ${ }^{2}$ Technische Universität München, Munich, Germany; \\ ${ }^{3}$ Universität zu Lübeck, Lübeck, Germany \\ Correspondence to: d.gola@uni-luebeck.de
}

Individual risk prediction based on genome-wide polygenic risk scores (PGRS) utilizing millions of genetic variants has attracted much attention. It is under debate whether PGRS models can be applied -without loss of precision -to populations of similar ethnic but different geographical background than the one the scores were trained on. Here, we examine how PGRS trained in one population-specific but European data set perform in other European subpopulations. Using data from UK and Estonian biobanks as well as case-control data from the German population we show that PGRS have the highest performance in their corresponding population testing data sets, whereas their performance significantly drops if applied to testing data sets from different European populations. This result has direct impact on the clinical 
usability of PGRS for risk prediction models utilizing PGRS: a population effect must be kept in mind when applying risk estimation models which are based on additional genetic information even for individuals from different European populations of the same ethnicity.

\section{A Consistent Estimator of Kinship and a General Definition of Heritability in Non-Random Mating Populations}

Goudet $J^{1}$, Weir $B^{2}$

${ }^{1}$ University of Lausanne, Lausanne, Switzerland; ${ }^{2}$ University of Washington, Seattle, WA, USA

Correspondence to: jerome.goudet@unil.ch

Kinship, or relatedness, is a central concept in many areas of biology and genetics. In quantitative and human genetics, it is the essential element in the animal model, a mixed model used to infer heritability and carry out Genome Wide Association Studies, as it captures how dependent the observations are. We are particularly interested in the understudied populations with mixed ancestries. Using the 1000 genome consortium data, we show beta, an estimator of kinship based on genome wide similarity (Weir and Goudet, Genetics, 2017), to be consistent, in particular in admixed populations. The standard definition of kinship based on the correlation or covariance coefficients is inconsistent for admixed individuals. We identify the (simple) relation between beta and the covariance based estimator, and show the two matrices have the same trace and share most of their eigenvalues. We further establish that the relation between heritability and the genetic and environmental variance components must account for both inbreeding and kinship. Last, using simulated traits with varying architecture on the 1000 genome dataset, we show that heritability estimates with beta or the covariance definition as kinship are unbiased and have small variances, whereas heritability estimated with the correlation definition are biased.

\section{Evaluating Short-Read Whole-Genome Sequencing Accuracy through Pseudo-Replication}

Herzig A, Velo区Suárez L, Le Folgoc G, Génin E

UMR 1078, Inserm, Paris, France

Correspondence to: anthony.herzig@inserm.fr

Current short-read based sequencing technologies give largely accurate but imperfect approximations of true human genomes. Sequencing errors are frequent and occur at prevalences that depend on the genomic context and on the sequencing and bioinformatics tools used. Quantifying the accuracy of whole-genome sequencing (WGS) data is necessary to evaluate results obtained in many applications but it is problematic in the absence of the hidden true genomes against which to compare. Only a small number of gold-standard genomes have been laboriously cataloged, such as those presented by the Genome in a Bottle project.
Technical replication studies are informative for understanding sequencing error-rates yet lack interpretability in the presence of batch effects. Here, we approach the problem by creating pseudo-replicates performed via a simple partitioning of raw unaligned read data into two groups. Thus creating a pair of variant call-sets from a single DNA sample, sequenced at half the depth, which can be contrasted against each other to give insights into experimental repeatability, variations in sequencing accuracy across the genome, and the efficacy of quality control measures.

We demonstrate our method on data from the GAZEL-ADN pilot project; a group of individuals with WGS data derived once from blood and once from saliva. This study allows us to present the utility of pseudo-replication, with the technique granting us the conclusion of equivalent quality between saliva- and bloodbased WGS that could not be established from only analyzing the original data.

\section{Improved Power and Precision with Whole Genome Sequencing Data in Genome-Wide Association Studies of Inflammatory Biomarkers}

Höglund J, Rafati N, Rask-Andersen M, Enroth S, Karlsson T, Ek W, Johansson $\AA$

Uppsala University, Uppsala, Sweden

Correspondence to: julia.hoglund@igp.uu.se

Genome-wide association studies (GWAS) have identified associations between thousands of common genetic variants and human traits. However, common variants usually explain a limited fraction of the heritability of a trait. A powerful resource for identifying trait-associated variants is whole genome sequencing (WGS) data in cohorts comprised of families or individuals from a limited geographical area. To evaluate the power of WGS compared to imputations, we performed GWAS on WGS data for 72 inflammatory biomarkers, in a kinship-structured cohort. When using WGS data, we identified 18 novel associations that were not detected when analyzing the same biomarkers with genotyped or imputed SNPs. Five of the novel top variants were low frequency variants with a minor allele frequency (MAF) of $<5 \%$. Our results suggest that, even when applying a GWAS approach, we gain power and precision using WGS data, presumably due to more accurate determination of genotypes. To infer causality, a two-stage design Mendelian Randomization (MR) was performed on the biomarkers that had colocalized signals with either eQTLs in blood or with inflammatory diseases in previously published association studies. IL-12B, that colocalized with results for previous associations with psoriasis and ulcerative colitis, showed a causal effect on psoriatic arthritis. IL-15RA, that colocalized with eQTLs in blood, showed a causal effect on eczema. As some causal effects can be seen, this, together with a more accurate determination of genotypes, can aid in the characterization of the genetic architecture of biomarker level variability in relation to the direction of effect on disease outcome. 


\section{Application of Bayesian Networks to Rheumatoid Arthritis and Intermediate Biological Marker Data}

Howey $R$, Cordell $H$

Newcastle University, Newcastle, UK

Correspondence to: richard.howey@ncl.ac.uk

There is an increasing interest in using causal analysis methods to move beyond initial analysis strategies, such as those applied in the context of genome wide association studies. One approach is to use Bayesian Networks (BN) which allow the relationships between biological and phenotypic data to be investigated in an exploratory manner, and are particularly suited to data sets with many variables. We consider a recent study of rheumatoid arthritis (RA) and the possible influences of methylation and gene expression in CD4+ T cells and B cells. In this study the causal inference test (CIT) was applied using many candidate variable triplets consisting of SNP, methylation and DNA expression data, focussing on SNPs with prior evidence of genetic association with RA. The results from this study suggested many genes where DNA methylation may mediate RA genetic risk. We applied BN analysis to the same dataset, exploring the candidate variable triplets as well as additional individual data for sex, age and RA status. We also investigated the benefit of modelling multiple variables simultaneously in a large, complex network. Our results showed replication of only some of the findings of the original study. This is not surprising as analytic methods have different assumptions and may be affected by different features of the data. This example highlights the benefit of exploring different analysis methods even for the same data set.

\section{Survival of the Densest Explains the Expansion of Mitochondrial Deletions in Skeletal Muscle Fibres}

\author{
Insalata $F^{1}$, Hoitzing $H^{1}$, Aryaman $\mathcal{J}^{2}$, Jones $N^{1}$ \\ ${ }^{1}$ Imperial College London, London, UK; ${ }^{2}$ University of Cambridge, \\ Cambridge, UK \\ Correspondence to: f.insalata17@imperial.ac.uk
}

The expansion of deleted mitochondrial DNA (mtDNA) molecules has been linked to ageing, particularly in skeletal muscle fibres. Despite three decades of research, the mechanism underlying this phenomenon has remained unclear. Previous accounts have assigned a selective advantage to the mitochondrial deletions, but, in fact, cells can selectively remove defective mtDNA. Justified by our microscopic understanding of mtDNA genetics, we introduce the mechanism of stochastic survival of the densest, adding noise and spatial structure to the well-known generalised LotkaVolterra model. Our physically motivated model reproduces the expansion of deletions without free parameters based on the enhanced carrying capacity of mutants, notably even if they are assigned a selective disadvantage. We establish that the expansion takes place in a wave-like fashion and provide a functional form for the wavespeed that predicts that it drops with copy number, in agreement with experimental data. This functional form suggests the relevance of existing drugs for slowing the waves of mutants.
Our model is approximated by a reaction-diffusion system whose reaction term stems from the combination of noise and increased mutant carrying capacity. In parallel, we show that a standard model based on a replicative advantage for mutants cannot reproduce the features of the expansion. We show how survival of the densest can also account for the evolution of altruism and conclude by proposing skeletal muscle ageing as a candidate exemplar for the role of noise and spatial structure in yielding novel evolutionary phenomena.

\section{Sibling Difference Analyses Reveal Polygenic Risk Score Confounding}

Joshi $P^{1}$, Timmer $P^{2}$, Clark $D^{2}$, Wilson $\mathcal{J}^{2}$

${ }^{1}$ Joshi, Edinburgh, UK; ${ }^{2}$ University of Edinburgh, Edinburgh, UK

Correspondence to: peter.joshi@ed.ac.uk

Polygenic risk scores are increasingly discussed for predicting disease risk and phenotypes(1), but confounding may still be present. Analysis of the effects of polygenic risk scores within sibling pairs (PRSsib) cannot plausibly be confounded by any postulated confounder we are aware of. At the same time, commercial ventures have started looking into in vitro genetic embryo selection amongst sibling embryos (PIGS (3)). PRSsib analysis can thus disentangle causality, confounding and genetic nurture, and reveal the magnitude of plausible effects of PIGS.

Published independent summary statistics were used to analyse the effect of PRSsib in siblings from UK Biobank, and to see the possible effect of PIGS if the higher PRS of two sibs had been selected for.

We find the relative effect(ratio/se) of PRSsib as against PRS on the selected outcome is $95 \% / 5 \%$ for height and $103 \% / 4 \%$ for LDL , but $47 \% / 4 \%$ for educational attainment (EA). In this sample, PIGS using these PRS and two siblings would have increased height/EA by $\{$ mean $(\mathrm{se})\} 0.8 \mathrm{~cm}(0.1) / 0.0$ years $(.02)$.

Embyronic screening for complex, especially social, traits appears ineffective.

\section{References}

1. Khera AV, Chaffin M, Aragam KG, Haas ME, Roselli C, Choi SH, et al. Genome-wide polygenic scores for common diseases identify individuals with risk equivalent to monogenic mutations. Nat Genet. 2018 Sep;50(9):1219-24.

2. Kong A, Thorleifsson G, Frigge ML, Vilhjalmsson BJ, Young AI, Thorgeirsson TE, et al. The nature of nurture: Effects of parental genotypes. Science. 2018 Jan 26;359(6374):424-8.

3. Polygenic Risk Scores and Genomic Prediction: Q\&A with Stephen Hsu GEN - Genetic Engineering and Biotechnology News 


\section{ImputeSCOPA: a Fast, Random Forest-Based Phenotype Imputation Tool for Large-Scale Studies}

Kaakinen $M^{2}$, Anasanti $M^{1}$, Jarvelin $M-R^{1}$, Prokopenko $P^{2}$

${ }^{1}$ Imperial College London, London, UK; ${ }^{2}$ University of Surrey, Guildford, UK

Correspondence to: m.kaakinen@surrey.ac.uk

Missing data are ubiquitous but often ignored, leading to loss of power and biased parameter estimates. We investigated properties of missing data imputation methods within multi-phenotype genome-wide association studies (MP-GWAS), focussing on single and multiple-imputation (SI/MI) using Bayesian approach (MICE) and expectation-maximisation bootstrapping (EMB), $\mathrm{k}$-nearest neighbour $(\mathrm{kNN})$, left-censored imputation method (QRILC) and random forest (RF). We simulated genetic data for 5,000/50,000/500,000 individuals using Hapgen2, and highly $(\mathrm{r}=0.64)$ and moderately correlated $(\mathrm{r}=0.33)$ phenotypes $(3 / 9 / 30 / 120)$ for them. We randomly selected common, low-frequency and rare variants to be significantly $\left(P<5 \times 10^{-8}\right)$ associated with the simulated phenotypes. We considered several proportions of missing data (1/5/20/50\%) under missing completely at random (MCAR), missing at random (MAR), and missing not at random (MNAR). We used the Root Mean Squared Error (RMSE) for evaluating the estimates from imputed vs. full data analysis. RF and MI-EMB had the least biased estimates under MCAR. RF also outperformed under MAR, whereas QRILC outperformed under MNAR. RF was applied to the Northern Finland Birth Cohorts (NFBC) 1966 and $1986(\mathrm{~N}=4,955$ and $\mathrm{N}=2,687$, respectively) for the imputation of anthropometric and glycaemic measurements and 149 serum metabolite levels. MP-GWAS of 31 amino acids showed a novel association at ADAMTS after imputation with RF $\left(P=2.61 \times 10^{-11}\right.$ vs. $P=5.68 \times 10^{-7}$ in complete case analysis $)$ and improved power at FCGR $3 B\left(\mathrm{P}=1.86 \times 10^{-9}\right.$ vs. $\left.P=1.72 \times 10^{-8}\right)$. We implemented RF into a user-friendly and computationally efficient imputeSCOPA software which is eight times faster than any currently available phenotype imputation software and is applicable to large-scale datasets such as the UK Biobank.

Funding: H2020-SC1-HBC-28-2019-LONGITOOLS.

\section{Visceral Adiposity and Its Impact on Type 2 Diabetes Development - The "Rise-And-Fall" of a Causal Effect} Karlsson $T^{1}$, Schmitz $D^{1}$, Ek $W^{1}$, Rask-Andersen $M^{1}$, Johansson $\AA^{1}$

Uppsala University Uppsala, Sweden

Correspondence to: torgny.karlsson@igp.uu.se

Visceral adipose tissue (VAT) -or deep belly fat -is known to be associated with an increased risk of a number of cardiometabolic diseases, including type 2 diabetes. Using non-linear prediction models based on either Dual-energy X-ray absorptiometry (DXA) or Magnetic resonance imaging (MRI) data of visceral adiposity, we predicted the $\operatorname{VAT}_{\text {mass }}\left(\mathrm{VAT}^{\wedge}\right)$ in over 325,000 white British individuals from the UK Biobank. In a recent study, we found a strong causal relationship between $\mathrm{VAT}^{\wedge}$ and the risk of type 2 diabetes, an effect that was significantly larger in females compared to males. We also showed that $\mathrm{VAT}^{\wedge}$ was associated with diabetes risk in a non-linear manner, for which, however, a causal relationship could not be established. Here, we perform non-linear Mendelian randomization (MR), using a stratification technique called the localized average causal effect. We find a strong non-linear causal effect of $\mathrm{VAT}^{\wedge}$ on type 2 diabetes, both in females (Cochran's $\left.Q: p=7.4^{\star} 10^{-4}\right)$ and in males $\left(p=7.1^{\star} 10^{-10}\right)$, such that the non-constant odds ratio exhibits a highly prominent peak at intermediate depots of $\mathrm{VAT}^{\wedge}$, only to decrease again at high $\mathrm{VAT}^{\wedge}$. We also show that this behavior cannot exclusively be a consequence of participation bias, where a larger drop-out of diseased individuals with high $\mathrm{VAT}^{\wedge}$ would result in a non-representative sampling of the true exposure-outcome association.

The research was funded by SSMF, VR, and SciLifeLab, as well as Kjell and Märta Beijers, Göran Gustafssons, Borgström/ Hedström, Åke Wiberg, the Swedish Heart-Lung, and the Swedish Diabetes Foundations.

\section{Incorporating and Validating the Impact of Priors on DNA Prediction of External Visible Characteristics}

\author{
Katsara $M-A^{6}$, Nothnagel $M^{5}$, Branicki $W^{3}$, Pospiech $E^{3}$, \\ Kayser $M^{1}$, Walsh $S^{2}$, Hysi $P^{4}$ \\ ${ }^{1}$ Erasmus MC University Medical Centre Rotterdam - Department \\ of Genetic Identification, Rotterdam, The Netherlands; ${ }^{2}$ Indiana \\ University/Purdue University - Department of Biology, West \\ Lafayette, IN, USA; ${ }^{3}$ Jagellonian University - Malopolska Centre \\ of Biotechnology, Krakow, Poland; ${ }^{4}$ St Thomas Hospital - \\ Department of Twin Research \& Genetic Epidemiology, London, \\ UK; ${ }^{5}$ University of Cologne - Cologne Center for Genomics, \\ Cologne, Germany; ${ }^{6}$ University of Cologne - Cologne Center for \\ Genomics, Cologne, Germany \\ Correspondence to: mkatsara@uni-koeln.de
}

Predicting externally visible characteristics (EVCs) from genetic data, often referred to as Forensic DNA Phenotyping, has raised major interest in forensic genetics over the last years. Several studies have recently developed and forensically validated predictive tools comprising of labtools and statistical tools, for traits such as eye, hair and skin color. Using prior information, e.g. obtained from the trait prevalence across geographic regions or populations, may potentially improve prediction accuracy, but has not been investigated thus far. Here, we performed a systematic assessment of the impact of incorporating prior values on the prediction for a number of EVCs, including eye, hair and skin color, hair structure and freckles, with respect to commonly used performance measures. We also compared the performance of the priorincorporating model to that of the prior-free model. We show that prediction is affected to a different degree for the different EVCs but also single EVC categories. We further show that, although priors have the potential to increase prediction performance, misspecification of prior values can lead to dramatic losses in overall accuracy. Our results emphasize the importance of a precise specification of priors in order to achieve valid and accurate results. This research has received funding from the EU Horizon 2020 VISAGE project (no. 740580). 


\section{A Comprehensive Transcriptional Map of Knee Osteoarthritis}

Katsoula $G^{1}$, Southam $L^{1}$, Steinberg ${ }^{1,2}$, Wilkinson $J^{3}$, Zeggini $E^{1}$

${ }^{1}$ Institute of Translational Genomics, Helmholtz Zentrum

München, Munich, Germany; ${ }^{2}$ Cancer Research Division, Cancer

Council NSW, Sydney, NSW, Australia; ${ }^{3}$ Department of Oncology

\& Metabolism, University of Sheffield, Sheffield, UK

Correspondence to: georgia.katsoula@helmholtz-muenchen.de

Osteoarthritis is a degenerative joint disease with huge public health burden and no curative therapy. Therefore, it is urgent to translate insights from genomics into disease mechanisms. We molecularly endotype joint tissues from 200 patients undergoing total knee replacement.

We generated RNA-seq data from patient knee tissues (primary chondrocytes from low-grade and high-grade cartilage, synoviocytes, and adipocytes) and genotype data from peripheral blood. We: (1) investigate gene expression differences between low and high-grade cartilage (2) examine alterations in chondrocyte population markers and (3) determine expression quantitative loci (eQTL) effects in all the aforementioned tissues.

Differential expression analysis identified 4,512 and 4,365 significantly upregulated and downregulated genes in high-grade cartilage respectively. Strong effects were observed for genes associated with osteoarthritis pathology including CRLF1 $\left(\mathrm{P}=1.54 \mathrm{e}^{-86}\right.$, $\mathrm{LFC}>2)$ and $C H R D L 2,\left(\mathrm{P}=9.19 \mathrm{e}^{-64}, \mathrm{LFC}<-2\right)$. Functional analyses pointed significantly enriched terms including skeletal system development $\left(\mathrm{P}=3.49 \mathrm{e}^{-06}\right)$, ossification $\left(\mathrm{P}=1.48 \mathrm{e}^{-05}\right)$ and activation of ECM-receptor interaction pathway $\left(\mathrm{P}=4.14 \mathrm{e}^{-06}\right)$. We detected significant downregulation in the expression of effector chondrocytes markers in high-grade cartilage, a chondrocyte population associated with high metabolic activity in early-stage osteoarthritis. The eQTL analysis revealed widespread significant associations between genetic variants and gene expression in all tissues.

We have identified large-scale transcriptional signatures of disease grade in key tissues of the knee joint in the largest osteoarthritis transcriptomic analysis to date. The identified genes and eQTL forward the understanding of molecular mechanisms in osteoarthritis. The impacted pathways serve as potential targets for novel therapeutics.

\section{Exploring and Improving Deep Learning Methods for Genomic Prediction}

\author{
Kelly C, Gaffney A, McLaughlin R \\ Trinity College Dublin, Dublin, Ireland \\ Correspondence to: oceallc@tcd.ie
}

Recent improvements in polygenic prediction have received much attention; however, the explained variance for all traits remains stubbornly lower than the theoretical maximum as represented by its estimated heritability. Deep Learning using artificial neural networks (ANNs) has also been gaining traction due to its widespread success in generating accurate prediction models from complex input data such as audio and image information. It is of current interest to explore whether this technique improves upon standard prediction methods employed today.

The discrepancy between heritability and explained variance may partially be due to the strictly linear methods employed by conventional GWAS and polygenic risk score (PRS) calculation. As it is well established that complex non-linear interactions are ubiquitous in determining the outcome of biological processes, it is reasonable to suspect that some of the variation of a trait is as a result of these epistatic interactions that are not well modelled by the methods currently employed in genetic prediction.

Previously, the major confounding effects of population structure and how to account for them in such a non-linear framework have not been actively explored. Here we have investigated genomic prediction strategies in the context of population structure in the 1001 Arabadopsis genomes project and a large European GWAS for amyotrophic lateral sclerosis (ALS). Variance in Arabadopsis flowering time explained by a trained ANN is significantly higher than from PRS ( $22=0.714$ vs. 0.198). In ALS, we show that our estimates of variance explained are confounded, necessitating strategies to adequately control the effects of pervasive structure in human populations.

\section{Predicting Risk of Neuropsychiatric Disorders Using Genotypes and Neural Networks}

Lysenkov V, Khan S, Elo L

University of Turku, Turku, Finland

Correspondence to: sofia.khan@utu.fi

In recent years, the accurate detection of known single nucleotide variants (SNVs) has evolved into a clinical routine. Further, the discovery of novel SNVs may lead to their functional annotation and phenotypic association. Together with their metadata and clinical significance, the information provides insight into the genetic architectures of diseases.

To date, a number of studies have implemented statistical and machine learning methods to generate predictive risk scores from genotype data. However, thus far prediction of a phenotypic trait from genotype data for a particular individual in the context of multifactorial diseases has remained challenging. Limiting factors include increased complexity due to a large variant space putatively explaining the phenotype in every sample, and the consequent need for nonlinear feature selection, as well as the limited availability of appropriate datasets. This study appraises the existing approaches and aims to determine whether the gap can be mitigated at all.

We leverage variants called from the whole exome sequencing of several thousand case subjects having a neuropsychiatric disorder, balanced by the controls. We describe the properties and the preparation of the data, as well as the modelling aspects, using the development of a convolutional neural network classifier as a practical example. Thereby, we estimate the challenges of the domain and demonstrate the practicalities of applying deep learning methods to the genotype data. 


\section{Martingale Residual Based Approach for Cox Modeling from High-Dimensional Data}

Kolde $A^{4}$, Käärik $M^{1}$, Fischer $K^{1}$, Joshi $P^{2}$, Esko $T^{3}$

${ }^{1}$ Institute of Mathematics and Statistics, University of Tartu,

Tartu, Estonia; ${ }^{2}$ Centre for Global Health Research, Usher Institute of Population Health Sciences and Informatics, University of Edinburgh, UK; ${ }^{3}$ Estonian Genome Center, Institute of Genomics, University of Tartu, Tartu, Estonia; ${ }^{4}$ Institute of Genomics,

University of Tartu, Tartu, Estonia

Correspondence to: anastassia.kolde@ut.ee

Survival modelling is a natural approach to study genetic associations to many phenotypes and it has been shown to be more sensitive than traditional regression based methods (Hughel et al., 2019). Still, it is not commonly used in Genome Wide Association Studies (GWAS) due to prohibitive computational cost. The Cox proportional hazard model can be approximated with linear regression by converting the survival trait to martingale residuals, thus enabling the use of common GWAS tools. This approach has been taken in many papers (Joshi et al., 2016), however, it is known that, theoretically, the approximation only works within certain bounds (Thernau et al., 1990).

The goal of this work is to explore the bounds theoretically and experimentally through simulations to determine if and when the approximation is usable within GWAS and other omics data analysis settings.

\section{References}

Hughey, J.J., Rhoades, S.D., Fu, D.Y., Bastarache, L., Denny, J.C. and Chen, Q., 2019. Cox regression increases power to detect genotype-phenotype associations in genomic studies using the electronic health record. BMC genomics, 20(1), pp.1-7.

Joshi, P.K., Fischer, K., Schraut, K.E., Campbell, H., Esko, T. and Wilson, J.F., 2016. Variants near CHRNA3/5 and APOE have age-and sex-related effects on human lifespan. Nature communications, 7(1), pp.1-7.

Therneau, T.M., Grambsch, P.M. and Fleming, T.R., 1990. Martingale-based residuals for survival models. Biometrika, 77(1), pp.147-160.

\section{A Genome-Wide Association Study of the Age-of-Onset of Idiopathic Pulmonary Fibrosis Using Time-to-Event Analysis Methods}

\author{
Kraven $L^{5}$, Allen $R^{5}$, Taylor $A^{1}$, Oballa $E^{1}$, Kang'ombe $A^{1}$, \\ Kaminski $N^{7}$, Whyte $M^{4}$, Tobin $M^{5}$, Sayers $I^{6}$, Hubbard $R^{6}$, Fahy $W^{1}$, \\ Maher $T^{2}$, Molyneaux $P^{2}$, Navaratnam $V^{3}$, Saini $G^{3}$, Yeo $A^{1}$, \\ Jenkins $G^{6}$, Wain $L^{5}$ \\ ${ }^{1}$ GlaxoSmithKline, London, UK; ${ }^{2}$ Imperial College London, \\ London, UK; ${ }^{3}$ Royal Brompton Hospital, London, UK; ${ }^{4}$ University \\ of Edinburgh, Edinburgh, UK; ${ }^{5}$ University of Leicester, Leicester, \\ UK; ${ }^{6}$ University of Nottingham, Nottingham, UK; ${ }^{7}$ Yale University, \\ New Haven, CT, USA \\ Correspondence to:Ik188@le.ac.uk
}

Rationale: Idiopathic pulmonary fibrosis (IPF) is characterised by progressive scarring of the lungs and poor prognosis. Previous genome-wide association studies (GWAS) have identified 14 genetic signals associated with IPF susceptibility but none have investigated the genetic determinants of age-of-onset, which could improve our understanding of underlying mechanisms and identify new drug targets.

To perform the first GWAS for age-of-onset in IPF.

Individuals with IPF from three studies were included: PROFILE ( $n=465)$, Trent Lung Fibrosis $(n=210)$ and UK Biobank $(n=98)$. We used either a subject's age-at-diagnosis or age-at-enrolment into their study as a proxy for their age-of-onset. We applied a Cox proportional-hazards model, adjusting for sex, smoking history and the first 10 genetic principal components. All variants with $\mathrm{P}<0.05$ in each study and that had a consistent direction of effects across all three were meta-analysed.

$7,179,468$ genetic variants passed quality control in all three studies. Of these, 248 passed the criteria above and were metaanalysed. No variants reached genome-wide significance $\left(\mathrm{P}_{\text {meta }}<5 \times 10^{-8}\right)$, but five independent genetic signals were suggestively significant $\left(\mathrm{P}_{\text {meta }}<5 \times 10^{-6}\right)$. The most significantly associated variant was rs183759512, located on chromosome 7 within an intron of DOCK4, where each copy of the risk allele corresponded to a younger age-of-onset $(\mathrm{HR}=2.20,95 \% \mathrm{CI}=[1.60,3.02]$, $\mathrm{P}_{\text {meta }}=1.1 \times 10^{-6}$ ).

We identified five signals of association with age-of-onset for IPF of potential interest that require further investigation. We will seek additional support for these novel signals in independent data sets.

\section{GWAS of Transferrin N-Glycans: One Step Closer to Understanding the Genetics of Protein Glycosylation}

\author{
Landini $A^{5}$, Navarro $P^{4}$, Trbojevic-Akmacic $P^{2}$, Vuckovic $F^{2}$, \\ Hayward $C^{4}$, Lauc $G^{3}$, Klaric $L^{4}$, Wilson $J^{1}$ \\ ${ }^{1}$ Centre for Global Health Research, Usher Institute, University \\ of Edinburgh, Edinburgh, UK; MRC Human Genetics Unit, \\ Institute of Genetics and Molecular Medicine, University of \\ Edinburgh, Edinburgh, UK; ${ }^{2}$ Genos Glycoscience Research \\ Laboratory, Zagreb, Croatia; ${ }^{3}$ Faculty of Pharmacy and \\ Biochemistry, University of Zagreb, Zagreb, Croatia; ${ }^{4} \mathrm{MRC}$ \\ Human Genetics Unit, Institute of Genetics and Molecular \\ Medicine, University of Edinburgh, Edinburgh, UK; ${ }^{5}$ University of \\ Edinburgh, Edinburgh, UK \\ Correspondence to: arianna.landini@ed.ac.uk
}

Glycomics, studying the collection of glycans in biological systems, is an emerging field among omics data. Despite glycans being involved in the aging process and in a wide variety of complex diseases, genetic regulation of glycosylation is yet not fully understood. To help address this knowledge gap, we carried out the first study integrating genomics and $\mathrm{N}$-glycomics of the blood plasma glycoprotein transferrin. We performed genome-wide association studies of 35 UPLC-quantified transferrin N-glycan traits in CROATIA-Korcula $(\mathrm{N}=948)$ and VIKING $(\mathrm{N}=959)$ cohorts, using Haplotype Reference Consortium imputed genotypes. GWAS results were used to perform meta-analysis and to replicate discovered loci. We identified 832 unique genome-wide significant associations $\left(\mathrm{P}<1.56 \times 10^{-9}\right)$ at 10 loci, mapping in genes encoding glycosyltransferases (MGAT5, FUT6, FUT8, ST3GAL4, 
B3GAT1), transcription factors regulating glycosylation and/or targeting a region in transferrin gene (HNF1A, FOXI1), genes related to glycosylation (MSR1) and transferrin $(T F)$, or previously associated with immunoglobulin G (IgG) N-glycosylation (NXPE1/NXPE4). 3 of these genomic regions (TF, MSR1, FOXI1) have not been previously associated with glycosylation, while 6 of them, found in CROATIA-Korcula, replicated in VIKING cohort (MGAT5, TF, ST3GAL4, B3GAT1, FUT8, FUT6). Our results contribute expanding the current knowledge about genetic regulation of $\mathrm{N}$-glycosylation and suggest that while some genes are involved in the N-glycosylation of both transferrin and IgG, others instead participate in N-glycosylation of only one of these proteins. In fact, an only partial overlap was noticed between genes we plausibly identified as involved in transferrin $\mathrm{N}$-glycosylation and those reported previously by IgG N-glycosylation association studies.

\section{Genome-Wide SNP-by-rs35705950 Interaction Analysis of Susceptibility to Idiopathic Pulmonary Fibrosis}

\author{
Leavy $O^{5}$, Allen $R^{5}$, Oldham $J^{4}$, Guillen-Guio $B^{3}$, Braybrooke $R^{6}$, \\ Hubbard $R^{6}$, Ma S- $F^{8}$, Fingerlin $T^{1}$, Kaminski $N^{9}$, Zhang $Y^{7}$, \\ Schwartz $D^{1}$, Maher $T^{2}$, Molyneaux $P^{2}$, Flores $C^{3}$, Noth $1^{8}$, \\ Jenkins $G^{6}$, Wain $L^{5}$ \\ ${ }^{1}$ National Jewish Health, Denver, CO, USA; ${ }^{2}$ Royal Brompton \\ Hospital, London, UK; ${ }^{3}$ Universidad de La Laguna, San Cristóbal \\ de La Laguna, Spain; ${ }^{4}$ University of California Davis, Davis, \\ CA, USA; ${ }^{5}$ University of Leicester, Leicester, UK; ${ }^{6}$ University \\ of Nottingham, Nottingham, UK; ${ }^{7}$ University of Pittsburgh, \\ Pittsburgh, PA, USA; ${ }^{8}$ University of Virginia, Charlottesville, VA, \\ USA; ${ }^{9}$ Yale School of Medicine, New Haven, CT, USA \\ Correspondence to: ocl3@leicester.ac.uk
}

Idiopathic pulmonary fibrosis (IPF) is a complex and heterogeneous fibrotic lung disease with median survival of 3 years. Genome-wide association studies (GWAS) have identified 14 genetic loci that influence IPF risk. A SNP, rs35705950, in the promoter region of the $M U C 5 B$ gene, is the largest known risk factor of IPF (odds ratio $=4.84$ [95\%CI: 4.37, 5.36], case minor allele frequency $=29.1 \%(\mathrm{~T}))$. We performed a genome-wide $\mathrm{SNP}^{\star}$ rs35705950 interaction analysis of IPF risk to understand the contribution of epistatic effects on IPF risk. Two different interaction meta-analyses were performed on up to 3,069 cases and 18,049 controls of European ancestry (three studies). We modelled rs35705950 as: i) continuous risk allele dosages for rs35705950, and ii) under dominant genetic model, where individuals were classified as $M U C 5 B$ positive if they carried at least one copy of the rs35705950 risk allele. Recognising limitations in statistical power, we defined suggestive significance as interaction signals with $P<1 \times 10^{-5}$, and consistent direction of effect with $P<0.05$ for association in all 3 studies. Eight signals were identified in the dosage analysis, of which 3 were also significant under the dominant model. Of these 3 signals, 2 had an opposite direction of effect in $M U C 5 B$ positive vs $M U C 5 B$ negative individuals and 1 was only observed to be associated with IPF risk in MUC5B positive individuals. Our analyses have identified SNPs whose effect on IPF risk is dependent on MUC5B genotype. These findings will help to provide further insight into different biological pathways that underlie fibrosis.

\section{Copy Number Variation Associations with Plasma Protein Levels in Estonian Population}

Lepamets $M^{1}$, Kalnapenkis $A^{1}$, Kals $M^{2}$, Mägi $R^{1}$, Esko $T^{1}$, Võsa $U^{1}$

${ }^{1}$ Estonian Genome Centre, University of Tartu, Tartu, Estonia;

${ }^{2}$ Institute for Molecular Medicine Finland (FIMM), University of Helsinki, Helsinki, Finland

Correspondence to: maarja.lepamets@ut.ee

Proteins are the primary functional units in human body serving as intermediaries between genetic variants and complex traits. Identifying variants acting as protein quantitative trait loci (pQTL) can help us gain novel insights about molecular mechanisms leading to those traits. While multiple studies have concentrated on SNP pQTLs and their overlap with corresponding expression QTLs (eQTLs), the aim of this project is to do the same for copy number variations (CNV).

In our analysis of 500 Estonian Biobank individuals we identified 12 unique genome-wide significant $(\mathrm{P}<1.1 \mathrm{e}-7)$ associations between CNVs and plasma protein levels. Seven of the associated CNVs were located within 500kbp from the transcription start site of the respective protein-coding gene (cis). The strongest cisassociation was between SULT1A1 and a CNV overlapping with the gene encoding the same protein $(\mathrm{P}=1.1 \mathrm{e}-20)$. Additionally, we identified five trans-associations. Four of them involved an intergenic deletion in 3q12.1 region, which decreased the levels of ICAM2 ( $\mathrm{P}=9.0 \mathrm{e}-30)$, FLT4 $(\mathrm{P}=1.5 \mathrm{e}-24)$, PDCD1LG2 ( $\mathrm{P}=3.0 \mathrm{e}-15)$ and IL1R1 $(\mathrm{P}=6.4 \mathrm{e}-8)$-all encoded from different chromosomes. Furthermore, we identified a trans-association between proinflammatory cytokine IL18 and a CNV overlapping with NAIP gene $(\mathrm{P}=2.6 \mathrm{e}-10)$, which is a sensor component for NLRC4 inflammasome.

Finally, we cross-referenced CNV pQTLs with CNV eQTLs that were identified from the same individuals. We found an overlap of three CNV-gene/protein pairs, possibly reflecting the distinct sources of gene expression in blood and protein expression in plasma. Altogether, our results emphasize the importance of structural variation on the genetic variability of plasma protein levels. 


\section{An Atlas of Pleiotropy for the Human Genome}

$\underline{L i T^{2}}$, Ning $Z^{1}$, Yang $Z^{2}$, Zhai $R^{2}$, Shen $X^{3}$

${ }^{1}$ Karolinska Institutet, Stockholm, Sweden; ${ }^{2}$ Sun Yat-sen

University, Guangdong, China; ${ }^{3}$ University of Edinburgh,

Edinburgh, UK

Correspondence to: liting67@mail2.sysu.edu.cn

Pleiotropy describes shared genetic basis across complex traits. As a ubiquitous phenomenon across the genome, the concept of pleiotropy has been studied for over 100 years. Understanding the magnitude of pleiotropy of different genomic loci, genes, and genetic variants is of fundamental importance for dissecting the complexity of genetic architecture. Nevertheless, in order to measure the level of pleiotropy for a given gene or genetic variant, a statistical significance threshold has to be applied for the quantification of the number of regulated phenotypes. On the other hand, widespread phenotypic correlations need to be properly accounted for. We introduce a novel threshold-free modeling technique to estimate the "pleiotropicity" of a gene or genetic variant. We show that for different pleiotropic scenarios, the pleiotropicity parameter can be unbiasedly identified even if the signal-to-noise ratio of the genetic effects are too small. We perform genome-wide evaluation of pleiotropicity and identify top pleiotropic loci such as $\mathrm{MHC}$ of the human genome. We also investigate the connection between linkage disequilibrium and pleiotropicity and provide useful functional insights. Integration of the estimated pleiotropicity and established GWAS summary statistics allow us to reveal more about the genetic basis of complex traits and diseases; For instance, our results show that the more complex genetic architecture of the phenotypes, the more their heritabilites are enriched in highly pleiotropic regions of the human genome.

\section{Genomic Architecture of 184 Plasma Proteins in 18,884 Individuals: the SCALLOP Consortium}

\author{
Macdonald-Dunlop $E^{22}$, Joshi $P^{3}$, Peters $J^{2}$, Folkersen $L^{19}$, \\ Ingelsson $E^{17}$, Timmers $P^{3}$, Michaelsson $K^{14}$, Gustafsson $S^{11}$, \\ Enroth $S^{9}$, Johansson $\AA^{9}$, Smith $G^{6}$, Zhernakova $D^{8}$, \\ Siegbahn $A^{10}$, Kalnapenkis $A^{15}$, Eriksson $N^{23}$, Wheeler $E^{20}$, \\ Fu $J^{8}$, Franke $L^{8}$, Hayward $C^{21}$, Wallentin $L^{1}$, Esko $T^{16}$, Zeggini $E^{18}$, \\ Teunissen $C^{7}$, Langenberg $C^{20}$, Hansson $O^{5}$, Eriksson $P^{12}$, \\ Gyllensten $U^{9}$, Butterworth $A^{2}$, Mälarstig $A^{13}$, Wilson $J^{4}$
}

${ }^{1}$ Department of Medical Sciences and Uppsala Clinical Research Center, Uppsala University, Uppsala, Sweden; ${ }^{2}$ Cardiovascular Epidemiology Unit, Department of Public Health and Primary Care, University of Cambridge, Cambridge, UK; ${ }^{3}$ Centre for Global Health Research, Usher Institute of Population Health Sciences and Informatics, University of Edinburgh, Edinburgh, UK; ${ }^{4}$ Centre for Global Health Research, Usher Institute of Population Health Sciences and Informatics, University of Edinburgh \& MRC Human Genetics Unit, Institute of Genetics and Molecular Medicine, University of Edinburgh, Edinburgh, UK; ${ }^{5}$ Clinical Memory Research Unit, Department of Clinical Sciences, Lund University \& Memory Clinic, Skåne University Hospital, Malmö, Sweden; ${ }^{6}$ Department of Cardiology, Clinical Sciences, Lund University, Lund, Sweden and Skåne University Hospita, Scania, Sweden; ${ }^{7}$ Department of Clinical Chemistry, Amsterdam Neuroscience, Amsterdam UMC, Vrije Universiteit Amsterdam, Amsterdam, The Netherlands; ${ }^{8}$ Department of Genetics, University Medical Center Groningen, University of Groningen, Groningen, The Netherlands; ${ }^{9}$ Department of Immunology, Genetics, and Pathology, Biomedical Center, Science for Life Laboratory (SciLifeLab) Uppsala, Uppsala University, Sweden;

${ }^{10}$ Department of Medical Sciences and Uppsala Clinical Research Center, Uppsala University, Uppsala, Sweden; ${ }^{11}$ Department of Medical Sciences, Molecular Epidemiology and Science for Life Laboratory, Uppsala University, Uppsala, Sweden; ${ }^{12}$ Department of Medicine, Karolinska Institutet, Stockholm, Sweden; ${ }^{13}$ Department of Medicine, Karolinska Institutet, \& Pfizer Inc, Stockholm, Sweden; ${ }^{14}$ Department of Surgical Sciences, Uppsala University, Uppsala, Sweden; ${ }^{15}$ Estonian Genome Center, University of Tartu, Tartu, Estonia; ${ }^{16}$ Estonian Genome Center, University of Tartu, Tartu, Estonia \& Broad Institute of MIT and Harvard, Cambridge, MA, USA; ${ }^{17}$ GlaxoSmithKline, San Francisco, CA, USA; ${ }^{18}$ Helmholtz Zentrum München, Munich, Germany; ${ }^{19}$ Institute of Biological Psychiatry, Copenhagen, Denmark; ${ }^{20} \mathrm{MRC}$ Epidemiology Unit, University of Cambridge, Cambridge, UK; ${ }^{21} \mathrm{MRC}$ Institute of Genetics and Molecular Medicine, University of Edinburgh, Edinburgh, UK; ${ }^{22}$ MRC Human Genetics Unit, Institute of Genetics and Molecular Medicine, University of Edinburgh, Edinburgh, UK; ${ }^{23}$ Uppsala Clinical Research Center, Uppsala University, Uppsala, Sweden Correspondence to: e.macdonald.dunlop@ed.ac.uk

Proteins are the fundamental building blocks of life and participate in all biological processes. Plasma proteins show great promise as novel disease biomarkers, and their genetic determinants also help unravel underlying networks and causal pathways, 
pointing to new drug targets. However, to date, discoveries have been limited due to small sample sizes. The SCALLOP consortium of 20 cohorts was established to discover protein quantitative trait loci (pQTLs) in a large combined sample. We used Olink proximity extension assays to quantitate the abundances of 184 plasma proteins (from the CVD II \&III panels) in up to 18,884 subjects.

Genome-wide association meta-analysis revealed 17,506 genome-wide significant SNPs $\left(\mathrm{P}<2.72 \times 10^{-10}\right)$ associated with at least one protein, including both cis- and trans-pQTLs. Contrary to findings from previous smaller individual studies, our increased sample size reveals the majority of proteins to have at least one pQTL.

We show how a subset of these pQTLs co-localise with eQTLs and disease-related traits. We also interrogate regulatory mechanisms and protein-protein interaction networks from our greatly expanded catalogue of trans-pQTLs. Using two-sample Mendelian randomisation we recapitulate known causal associations of proteins on disease, including PCSK9 on LDL cholesterol. Additionally, we report the novel causal effect of adrenomedullin (ADM) on Neuroticism.

\section{Integrating Transcriptomics and Proteomics Data into pathWAS Allows Prediction of Pathway Functionality}

May-Wilson S, Macdonald-Dunlop E, Wilson J, Pirastu N

University of Edinburgh, Edinburgh, UK

Correspondence to: s.may-wilson@ed.ac.uk

Rationale: Understanding complex traits, multifactorial disease and their underlying biology is a primary goal of genetics. GWAS has seen much success in the discovery of loci related to complex traits, however, it can be challenging to pinpoint causal SNPs and genes. One method to tackle this has been the incorporation eQTL data with GWAS loci to improve the power of discovery for causal genes: TWAS. This method has the limitation of only examining genes in isolation and not in the context of relevant biological pathways.

Method: Here we present a method in which we stratify transcribed genes by biological pathways. We combine polygenic scores (PRS) based on GTEx eQTLs in the PredictDB dataset, for genes into one overall pathway score. By then exploiting a defined and measured protein end-point, it is possible to estimate the relative contribution of each gene to pathway functionality. The PRS $_{\text {pathway }}$ were trained in the isolated cohort ORCADES using elastic net penalised regression and tested in in the Vis cohort.

Results: In the testing set the method was successful in predicting the protein end point in six instances, significant after Bonferroni correction. These pathways are primarily those related to infectious disease response, including NOD-like receptor signalling, with a predictor for the MAPK signalling pathway significant in a linear regression model.

Conclusion: Gene pathway scoring offers the prospect of more powerful and holistic analysis of GWAS results, with the potential to investigate and discover causal pathways for complex traits.

48th European Mathematical Genetics Meeting

\section{Using Offspring Genotype Instead of True Genotype for Survival Analysis}

Möls M, Mändul M, Fischer K

University of Tartu, Tartu, Estonia

Correspondence to: martm@ut.ee

As the follow-up time for biobanks is still relatively short, the number of observed events can be quite small, despite the impressive size of the overall cohort. Therefore, it has been proposed to use parental survival times as outcomes in such analyses, whereas offspring genotypes are used as covariates. However, estimating the genotype effect using offspring genotypes in Cox proportional hazards model will lead to biased estimates. This simple approach is also theoretically questionable as the proportional hazard model will not be a valid model for offspring genotypes if it holds for parental genotypes. We will demonstrate how one can modify the Cox proportional hazards model to be compatible with offspring genotypes. The modified model will also provide unbiased estimates for genotype effects (comparable to the results one gets if one would use the true genotypes in survival analysis).

The methodology will be illustrated using the Estonian Biobank data.

\section{Is Competition for Resource between Genes a Driving Force of the Omnigenic Architecture?}

Naret $O$

École Polytechnique Fédérale de Lausanne, Lausanne,

Switzerland

Correspondence to: olivier.naret@epfl.ch

The precise genetic architecture of complex traits remains largely unexplained. As an extreme example of polygenicity, it was estimated through genome-wide association studies (GWAS) that up to 100,000 independent genetic variants affect height. While some of the top associated variants could be mapped to meaningful biological genes and pathways, most of them fall in the regulatory regions of supposedly irrelevant genes. This leaves the bulk of the biological underpinning of height heritability unexplained. Relying on the observation that any gene can be associated with a trait as long as it is expressed in a relevant cell or tissue, Jonathan Pritchard and colleagues proposed the "omnigenic model", which classifies the genes impacting a complex phenotype in two categories: 1) a minority of core genes that are specific to the biology of the trait; 2) a majority of peripheral genes that are effective through trans-regulating effects on core genes.

We test here if competition for cellular resources during translation between core and peripheral genes could be a trans-regulating mechanism. First, we demonstrate that the competition has only a minor effect on individual protein level variance. Second, we highlight that the competition produces a weak negative covariance across the proteome potentiating its end effect on the phenotype. Finally, we show that peripheral genes can explain a fraction of the heritability if enough core genes are expressed with a 
nonnull mean effect size. Altogether, our results show that competition for resources can be a minor driver of the omnigenic architecture of extremely polygenic traits.

\section{Discovery, Estimation and Prediction Analysis Using a Bayesian Survival Model for Complex Traits}

\author{
Ojavee $S^{1}$, Trejo-Banos $D^{1}$, Patxot $M^{1}$, Fischer $K^{2}$, Kousathanas $A^{1}$, \\ Robinson $\mathrm{M}^{1}$ \\ ${ }^{1}$ University of Lausanne, Lausanne, Switzerland; ${ }^{2}$ University of \\ Tartu, Tartu, Estonia \\ Correspondence to: svenerik.ojavee@unil.ch
}

Time-to-event analysis using genotypic data may enable a better understanding of the mechanisms and underlying genetic architecture behind the onset and development of common complex disease. Typically, time-to-event data are not normally distributed, are right censored and in genomic studies, the number of covariates strongly exceeds the number of observations. Previous work has not adequately addressed these issues, potentially leading to underpowered models and biased effect size estimates.

Here, we propose a hierarchical Bayesian model which assumes that time-to-event has a Weibull distribution, handles sparsity with spike and slab variable selection and variance partitioning, considers right censoring, and yields estimates of the proportion of genetic variance explained (SNP heritability). Through a series of computational advances, the model can handle datasets of hundreds of thousands of people and millions of genetic markers by combining synchronous parallel Gibbs sampling and adaptive rejection sampling.

In simulations, our approach outperformed previous approaches and provided better genetic effect estimation. We then applied our model to UK Biobank data for a wide-range of traits, including time-to-death, time to cardiovascular disease and timeto-menopause. We achieved $68 \%$ higher SNP heritability estimates compared to previous results for both time-to-menopause and time-to-menarche (SNP heritabilities of 0.26 and 0.42 respectively).

Our general framework enables more accurate discovery and estimation of the survival-related genomic marker effects providing novel insight into the genetic architecture of any very largescale time-to-event data, and unprecedented statistical power to predict risk for medically important traits.

\section{A Comparison of Two Software Tools for Disease-Gene Prioritization for Family-Based Sequencing Studies}

\section{Ormond C, Corvin A, Heron E}

Trinity College Dublin, Dublin, Ireland

Correspondence to: cathal.ormond@tcd.ie

Previous research has implicated both rare and common variation as contributing towards complex disorders. Whole genome sequencing (WGS) is a powerful technique that allows for the examination of a wide spectrum of DNA variants to identify genes that may be related to disorders of interest. While family-based sequencing studies have traditionally relied on applying hard filters on specific variant metrics (e.g. allele frequency, CADD scores, etc), some tools seek to combine multiple levels of prior evidence to perform disease gene-prioritization. pVAAST (Hu 2014, Nat Biotechnol) combines a modified gene-based linkage analysis with variant functional prediction and allele frequency in a unified framework. PERCH (Feng 2017, Hum Mut) provides a Bayesian framework to integrate information from gene interaction networks, rare variant association analysis and a Bayesian form of linkage analysis. However, as both tools implement novel techniques, it is difficult to interpret their prioritization scores, or indeed to compare the resulting scores for each gene to each other or to standard methods.

Here, we examine WGS data from the Coriell Institute CEPH 1463 pedigree, together with control GWAS data. We estimate an empirical null distribution for the scores for each gene for both tools by randomizing the phenotype status of each sample. To select disease genes of interest, we consider variants in coding regions with high CADD scores that co-segregate with fixed phenotype patterns. This allows us to examine the strengths and weaknesses of each tool, and to identify which constituent methods are most contributing to the final scores.

\section{Conditional Estimation of the Contribution of Genomic Annotations to Common Complex Diseases}

\author{
Patxot M, Kousathanas A, Erik Ojavee S, Trejo Banos D, \\ R. Robinson $M$
}

Université de Lausanne, Lausanne, Switzerland ${ }^{1}$

Correspondence to: marion.patxotbertran@unil.ch

Despite efforts to estimate the contribution of genomic regions to common complex trait variation, the distribution of effect sizes across functional annotations remains unknown. Previous studies estimate enrichment across annotations in a series of follow-up analyses rather than utilizing functional information to assess enrichment conditional on the rest of the genome. Here, we introduce a scalable Bayesian model that utilizes genomic annotations and individual-level data to jointly estimate marker effects while accounting for LD. SNP effects for each annotation group are modelled as a series of normal distributions providing inference on the genetic architecture of genomic enrichment. Effect size distributions can be compared across annotations and the genetic variance for each group simultaneously quantified. In simulations we demonstrate our method gives unbiased estimates of annotation enrichment under extreme LD scenarios. We apply the model to height, body mass index (BMI), type-2-diabetes (T2D) and coronary artery disease (CAD) in the UK Biobank. We find a stark contrast between BMI and height: BMI being highly polygenic with tiny effect sizes located 10 to $1000 \mathrm{~kb}$ from exons whereas height shows a variety of effect sizes in coding and non-coding regions. T2D and CAD are highly polygenic and we also observe that promoter regions contribute less than $5 \%$ to the phenotypic variance of all four traits. This method provides a full quantification of genetic architectures underlying complex traits and determines which genomic regions are influential, improving disease risk prediction. 


\section{Minimum Variance Unbiased Estimator of Genomic Relatedness Matrices}

Perdry $H$

Université Paris-Saclay — Inserm CESP, Paris, France

Correspondance to: herve.perdry@université-paris-saclay.fr

Genomic relatedness matrices (GRM) are widely used in human genetics, in particular for principal components analysis (PCA). The first principal components (PC) based on GRMs allow the assessment of population structure, and also to take it into account in genome-wide association studies. GRMs are usually computed from a set of SNP in low linkage disequilibrium (LD), extracted from a dense set of SNPs; this operation is known as \&ldquo;LD thinning\&rdquo;. If this operation were omitted, and in presence of a large subset of SNPs in a region with strong LD (e.g. the HLA region), the first PCs might be influenced essentially by this region, instead of representing variations of frequencies across the whole genome. However, the LD thinning operation might be a too drastic solution to this problem, resulting in the loss of valuable information.

\section{Using Genetics to Disentangle the Complex Relationship between Food Choices and Health Status \\ Pirastu $N^{5}, M c D o n n e l l C^{5}$, Grzeszkowiak $E^{5}$, Mounier $N^{1}$, Imamura $F^{3}$, Day $F^{3}$, Zheng $J^{4}$, Taba $N^{2}$, Esko $T^{2}$, Joshi $P^{5}$, Fischer $K^{2}$, Ong $K^{3}$, Gaunt $T^{4}$, Kutalik $Z^{1}$, Perry ${ }^{3}$, Wilson $J^{5}$ \\ ${ }^{1}$ Center for Primary Care and Public Health, University of Lausanne, Lausanne, Switzerland; ${ }^{2}$ Estonian Genome Center, Institute of Genomics, University of Tartu, Tartu, Estonia; ${ }^{3} \mathrm{MRC}$ Epidemiology Unit, Institute of Metabolic Science, Cambridge Biomedical Campus, University of Cambridge School of Clinical Medicine, Cambridge, UK; ${ }^{4} \mathrm{MRC}$ Integrative Epidemiology Unit, Bristol Medical School, Bristol, UK; ${ }^{5}$ Usher Institute - The University of Edinburgh, Edinburgh, UK \\ Correspondence to: nicola.pirastu@ed.ac.uk}

Food choices are one of the most important factors influencing health, thus understanding their genetic determinants may shed light on important biological mechanisms underlying disease. However, nutritional epidemiology is plagued by biases and confounding, where for example, health status or education affect both behaviour and reporting of consumption. We thus applied a novel post-GWAS Mendelian Randomisation (MR)-based correction method to measure and adjust for the effect of health-related traits on food choice/reporting. We show that these risk factors and diseases account for up to $42 \%$ of the genetic variance of reported food intake in UK Biobank. Bias-corrected GWAS on 29 food consumption traits in up to 445,779 individuals identifies numerous robustly associated loci. Simulations and results from genes with known function, show that ratio between the corrected and raw results (CRR) can be used to distinguish loci directly influencing food choice, allowing selection of more valid instrumental variables for $\mathrm{MR}$ and better gene prioritisation for functional follow up.

We show that the use of the direct-effect only instruments can substantially change the results of MR analysis. The corrected results show numerous causal food health relationships shedding a new light on nutritional epidemiology. Our new framework is a powerful tool to circumvent bias and confounding in nutritional epidemiology and can be used in all those cases where the exposure and the outcome are mutually causal. Moreover, it is helpful to identify those genes which are influencing directly the outcome of interest guiding the prioritization for functional studies.

\section{Long-Sightedness (Hyperopia) and Its Effect on Educational Attainment: Linear and Non-Linear Mendelian Randomization Analyses \\ Plotnikov D' ${ }^{1}$, Guggenheim J', Williams $C^{3}$, UK Biobank Eye and Vision Consortium ${ }^{2}$ \\ ${ }^{1}$ Cardiff University, Cardiff, UK; ${ }^{2}$ UK Biobank, Manchester, UK; \\ ${ }^{3}$ University of Bristol, Bristol, UK \\ Correspondence to: plotnikovd@cardiff.ac.uk}

Past observational studies conflict regarding whether children with long-sightedness (hyperopia) have poorer educational outcomes. The causality of this relationship has not been investigated to date. The aim of the current study was to test for a (potentially non-linear) causal effect of hyperopia on educational attainment. Linear and non-linear Mendelian randomization (MR) analyses were carried out in a sample of 60,529 unrelated UK Biobank participants. MR estimates were obtained using 2-Stage least squares (2SLS) regression in the full sample or in 10 deciles stratified by residual spherical refractive error. A polygenic risk score for refractive error was used as an instrumental variable. As a sensitivity analysis, inverse variance weighted non-linear MR was carried out with 131 SNPs as instrumental variables. Statistical power was assessed using simulations. In the observational analysis, each additional diopter (D) of refractive error was associated with 7.8 weeks $(-0.15$ years, $95 \%$ CI -0.15 to $-0.14, p<2 e-16)$ less time in education. MR assuming a linear relationship yielded a causal effect estimate of -0.02 years/D (95\% CI -0.05 to $0.005, \mathrm{p}=0.10)$. Non-linear MR analysis estimated a causal effect within the range -0.15 to +0.08 years $/ D$, with evidence of heterogeneity across strata $\left(\mathrm{I}^{2}=58.8 \%\right.$; Cochran's $\mathrm{Q}=21.3, \mathrm{p}=0.01)$. The results of this study support the hypothesis that the causal relationship between refractive error and time spent in education is non-linear, but did not support the hypothesis that hyperopia is causal risk factor for educational attainment. 


\section{Exploring the Genetic Architecture of the Human Neurological Proteome Using Whole Genome Sequencing}

\author{
Png $G^{4}$, Barysenka $A^{5}$, Tsafantakis $E^{1}$, Karaleftheri $M^{3}$, Dedoussis $G^{2}$ \\ Gilly $A^{5}$, Zeggini $E^{5}$ \\ ${ }^{1}$ Anogia Medical Centre, Anogia, Greece; ${ }^{2}$ Department of \\ Nutrition and Dietetics, School of Health Science and Education, \\ Harokopio University of Athens, Athens, Greece; ${ }^{3}$ Echinos \\ Medical Centre, Echinos, Greece; ${ }^{4}$ Helmholtz Zentrum Munich, \\ Munich, Germany; ${ }^{5}$ Institute of Translational Genomics, \\ Helmholtz Zentrum München - German Research Center for \\ Environmental Health, Neuherberg, Germany \\ Correspondence to: grace.png@helmholtz-muenchen.de
}

The human proteome has a stronger genetic component compared to many complex diseases, making it a valuable resource of potential disease biomarkers and drug targets. This is especially so for highly polygenic neurological disorders whose mechanisms remain elusive. Here, we present the first sequence-based protein quantitative trait loci (pQTL) analysis of 92 neurological proteins. We perform a meta-analysis using deep whole-genome sequencing (WGS) data from two isolated Greek cohorts, MANOLIS (22.5x WGS; $\mathrm{N}=1,356)$ and Pomak (18.4x WGS; $\mathrm{N}=1,537)$. A total of 123 independently-associated variants in 84 loci reach studywide significance $\left(P<1.14 \times 10^{-10}\right)$ for 63 proteins, all of which are at least nominally significant $\left(P<3.78 \times 10^{-4}\right)$ and have the same direction of effect in both cohorts. To further elucidate the genetic architecture, independent variants were classified into 89 (72\%) cisand $34(28 \%)$ trans-acting pQTLs. Ten variants have consequences equal to or more severe than missense, and 33 overlap regulatory regions. We also discover variants that have previously been linked to psychiatric disorders. For example, an intronic trans-pQTL in the ITIH4 gene is associated with increased NEP levels ( $r$ 22239547; $P=1.19 \times 10^{-129}$; BETA=0.637983; $\mathrm{SE}=0.026328$ ), and is an established risk variant for schizophrenia and bipolar disorder. This analysis represents the largest and only WGS-based pQTL study of neurological proteins to date, delivering insight into the rare and common genetic variant landscape underlying the human neurological proteome and its connection to neurological diseases.

\section{Determining the Role of Gene Expression on Human Sexual Dimorphism \\ Porcu $E^{1}$, Claringbould $A^{2}$, Lepik $K^{3}$, Franke $L^{2}$, Santoni $F^{1}$, Reymond $A^{1}$, Kutalik $Z^{1}$ \\ ${ }^{1}$ University of Lausanne, Lausanne, Switzerland; ${ }^{2}$ University of Groningen, Groningen, The Netherlands; ${ }^{3}$ University of Tartu, Tartu, Estonia \\ Correspondence to: eleonora.porcu@unil.ch}

Despite the prevalence of many diseases differs between women and men, only few published genome-wide association studies (GWAS) have been performed in a sex-stratified manner.
Nowadays the molecular bases of sex associated differences in complex traits remain poorly understood. We hypothesized that given the marked causal involvement of gene expression levels in complex traits, sex-biased trait associations might be driven by sex-biased eQTLs. To challenge this assumption, we performed a genome-wide analysis of sex-specific whole blood RNA-seq eQTLs from 3,447 individuals. Amongst the pre-selected 9 million SNPgene pairs (based on sex-combined association), we identified 18 genes with significantly (FDR 5\%) different eQTL effects in men and women. PheWAS analyses for these 18 eGenes on $>700$ traits revealed that sex-biased eQTLs in CDIP1 and PSMD5 translate into sex-specific trait-associations for trunk predicted mass. However, such examples are sporadic and sex-specific expression regulation does not systematically propagate to high-level traits. Next, we applied a sex-specific transcriptome-wide Mendelian Randomization approach (TWMR) by combining sex-specific summary statistics for both eQTLs and complex traits and observed the presence of a compensatory effect downstream of gene expression, e.g. genes with stronger eQTLs in women have weaker woman-specific causal effects on complex traits. Finally, we show that the sexspecific GWAS associations are not driven by sex-biased eQTLs. Our findings suggest that sex-specific trait associations can rarely occur as a consequence of sex-specific gene expression regulation in whole blood, hence other omics data are necessary to better understand the genetic basis of sexual dimorphism.

\section{Epigenome-Wide Association Study of Longitudinal Changes in Blood Metabolite Levels from Young to Middle Adulthood}

\author{
Pupko ${ }^{3}$, Draisma $H^{3}$, Zudina $L^{3}$, Balkhiyarova $Z^{3}$, \\ Wielscher $M^{1}$, Ala-Korpela $M^{2}$, Sebert $S^{2}$, Järvelin $M-R^{1}$, \\ Kaakinen $\mathrm{M}^{3}$, Prokopenko $\mathrm{I}^{3}$
}

${ }^{1}$ Imperial College London, London, UK; ${ }^{2}$ University of Oulu, Oulu, Finland; ${ }^{3}$ University of Surrey, Guildford, UK

Correspondence to: i.pupko@surrey.ac.uk

Associations between metabolite levels and DNA methylation (DNAm) have been reported. However, the relationship between longitudinal changes in metabolite levels and differential DNAm is underexplored. We assessed associations between epigenomewide blood DNAm and changes in the levels of blood proton nuclear magnetic resonance metabolomics-based traits. For 595 non-diabetic individuals from the Northern Finland Birth Cohort 1966 with metabolomics data at ages 31 (T1) and 46 years (T2) as well as concurrent DNAm data (T2), we calculated the average change in level per year between T1 and T2 for each of the 228 metabolomics-based variables. We used our methylSCOPA software for fast single-predictor longitudinal epigenome-wide association study (EWAS). We regressed out participant sex from the change in metabolomic variable level and used the resulting residuals as predictors of DNAm in EWAS for 832,569 markers on the Illumina (San Diego, CA, USA) MethylationEPIC BeadChip. We quality-controlled, residualized, and normalized the DNAm data, mapped genomic locations to CGCh37/hg19, and adopted the Bonferroni-corrected $P$-value $=4.4 \times 10^{-10}$ threshold to denote epigenome- and metabolome-wide statistical significance 
of association between predictor and epigenetic mark. We detected an epigenome- and metabolome-wide significant association between DNAm at cg16904859 (chr1:206,849,995; $\beta=2.31$; $\mathrm{SE}=0.36 ; P=3.2 \times 10^{-10}$ ) and change over time in the level of triglycerides in chylomicrons and extremely large very low-density lipoprotein particles. Additionally, we identified 67 epigenome-wide significant $\left(P<1 \times 10^{-7}\right)$ predictor-epigenetic mark associations, involving 28 unique epigenetic markers and 53 unique metabolomics-based variables. Using methylSCOPA, we detected associations between longitudinal changes in blood metabolite levels and DNAm.

Funding: H2020-SC1-2019-874739, WCRF-2017/1641.

\section{A Genomic Meta-Analysis of 184 Neuroproteins and Their Implied Causality on Psychiatric Disorders}

Repetto $L^{2}$, Navarro $P^{2}$, Wilson ${ }^{2}$, Shen $X^{1}$

${ }^{1}$ Sun Yat-sen University, Guangzhou, China; ${ }^{2}$ The University of Edinburgh, Edinburgh, UK

Correspondence to: linda.repetto@ed.ac.uk

Protein quantitative trait loci (pQTL) are essential to study the molecular basis of complex diseases, as they provide insights on the role of genetic variation in determining protein levels that modulate an individual's metabolic state. We quantified 184 proteins involved in neurological processes using the Olink Neurology and Neuro-exploratory panels in 1070 individuals of the Orkney Complex Disease Study (ORCADES) with genotypic information. For each protein, we performed a GWAS looking for loci associated with protein levels, both in the proximity of the proteincoding gene (in cis) and distantly (in trans). We discovered 48cisand 59trans- pQTL for 95 neuroproteins. Enrichment analyses on the proteins levels of expression show that the neuroproteins with cis-pQTL display enriched expression in the brain. We then investigated the potential causal effect of protein level variation on psychiatric disorders, including major depression, schizophrenia, and bipolar disorder for the proteins with cis-pQTL. Using our pQTL study and summary-level data from a large 2018 GWAS for major depressive disorder (Wray et al., 2018), we discovered 12 additional loci associated with the disease. Two of these 12 loci were also discovered in a later 2019 GWAS meta-analysis for major depression (Howard et al., 2019), strengthening our novel results, that highlighted loci through neurological pQTL with potentially relevant roles in psychiatric disorders. With these promising results, we set up a SCALLOP meta-analysis of levels of the same proteins in seven cohorts with a maximum sample size of 12,000 individuals to reveal more biology underlying psychiatric diseases.

\section{Leveraging Naturally Occurring Genetic Variation to Disentangle the Effects of Multiple Risk Factors on Disease}

Richardson $T^{1}$, Sanderson $E^{1}$, Tilling $K^{1}$, Holmes $M^{2}$, Davey Smith $G^{1}$

${ }^{1} \mathrm{MRC}$ Integrative Epidemiology Unit, Bristol, UK; ${ }^{2} \mathrm{MRC}$

Population Health Research Unit, Oxford, UK

Correspondence to: Tom.G.Richardson@bristol.ac.uk

Differentiating between causal and correlated risk factors is of critical importance for disease prevention. Genetic variation inherited at birth is typically robust to confounding and reverse causation and can be harnessed to separate the effects of multiple risk factors on disease using an approach called multivariable Mendelian randomization.

We firstly demonstrate this by investigating the effects of various lipid traits on coronary heart disease (CHD). For example, when analysed independently HDL cholesterol appears to be protective towards $\mathrm{CHD}(\mathrm{OR}=0.80,95 \% \mathrm{CI}=0.75-0.86)$, but provided weak evidence of a direct effect when adjusting for other lipids $(\mathrm{OR}=0.91,95 \% \mathrm{CI}=0.74-1.12)$. In contrast, our results suggest that apolipoprotein $\mathrm{B}$ is the predominant trait which accounts for the aetiological relationship between lipids and $\mathrm{CHD}(\mathrm{OR}=1.68,95 \%$ $\mathrm{CI}=1.54-1.84$ ).

The effects of childhood and adult obesity on disease are likewise extremely challenging to disentangle in an observational setting. However, separating them using genetic variation provides evidence that childhood adiposity has an indirect effect on CHD and type 2 diabetes along the causal pathway via adult adiposity $(\mathrm{OR}=1.49,95 \% \mathrm{CI}=1.33-1.68$ and $\mathrm{OR}=2.32,95 \% \mathrm{CI}=1.76-3.05$ respectively). This suggests that the detrimental impact of childhood obesity on these outcomes is likely attributed to individuals remaining overweight into adulthood. Conversely, childhood adiposity appeared to have a more direct effect on breast cancer risk $(\mathrm{OR}=0.59,95 \% \mathrm{CI}=0.50-0.71)$, which we postulate is due to its influence on earlier timing of puberty and sex hormone levels.

The current wealth of phenotypically rich datasets provides an unprecedented opportunity to dissect disease pathways using Mendelian randomization.

\section{Fast Imputation of Low Coverage Sequencing Data from Very Large Reference Panels}

$\underline{\text { Rubinacci } S^{1}}$, Ribeiro $D^{1}$, Hofmeister $R^{1}$, Delaneau $O^{1}$

University of Lausanne, Lausanne, Switzerland

Correspondence to: simone.rubinacci@unil.ch

Low coverage whole-genome sequencing $(0.5 \mathrm{x}-1 \mathrm{x})$ followed by imputation has been shown to recapitulate the same signals and discover new variants compared to imputation of SNP arrays (Pasaniuc, 2012; Gilly, 2019). However, imputation methods are computationally expensive and large reference panels cannot be used due to model constraints.

We describe Low Coverage Caller (LCC), a method for genotype imputation of low coverage sequencing datasets. The model, 
based on a hidden Markov model (Li and Stephens, 2003), has two key features. First, a linear-time sampling algorithm for haplotype configurations. Second, it uses a procedure to reduce the state space by selecting a subset of highly confident haplotypes. This allows LCC to be efficient while leveraging information from very large reference panels of haplotypes.

We use high-coverage data from the 1000 Genome Project and run LCC and Beagle4.1 on down-sampled coverages in the range $0.1 \mathrm{x}-8.0 \mathrm{x}$. We also perform imputation on 35 different SNP array models using Beagle5.1. For all the experiments, we use the HRC as a reference panel. We show that our method is more accurate and orders of magnitudes faster than other low-coverage sequencing imputation methods. We also show that imputation from $0.5 \mathrm{x}$ and $0.8 \mathrm{x}$ outperforms imputation of Illumina Global Screening Array and Omni2.5, respectively. This is particularly true at extremely rare variants, where there is an accuracy boost of $\sim 20 \%$.

LCC has a limited computational overhead and outperforms standard imputation from SNP arrays, allowing large-scale association studies to be based on low coverage sequencing.

\section{A Global-Local Variational Approach for Detecting Hotspots in Molecular Quantitative Trait Locus Studies}

\section{Ruffieux H}

MRC Biostatistics Unit, University of Cambridge, Cambridge, UK Correspondence to: helene.ruffieux@mrc-bsu.cam.ac.uk

We tackle modelling and inference for variable selection in the context of molecular quantitative trait locus (QTL) studies. We focus on detecting hotspots, i.e., genetic variants which, by controlling remotely the levels of many gene products, may shape the architecture of the genome and initiate decisive functional mechanisms underlying disease endpoints.

Existing hierarchical regression approaches designed to model hotspots suffer from two limitations: their discrimination of hotspots is sensitive to the choice of top-level scale parameters for the propensity of predictors to be hotspots, and they do not scale to the dimensions of the predictor and response spaces encountered in QTL applications.

We address these shortcomings by introducing a flexible hierarchical regression framework that is tailored to the detection of hotspots and scalable for the current QTL studies. Our novel framework allows information-sharing across outcomes and variants, thereby enhancing the detection of weak effects, and directly controls the hotspot propensity via a dedicated top-level representation. In particular, it implements a fully Bayesian model for hotspots based on the horseshoe shrinkage prior: its global-local formulation shrinks noise globally and hence accommodates the highly sparse nature of genetic analyses, while being robust to individual signals, thus leaving the effects of hotspots unshrunk. Inference is carried out using a fast variational algorithm coupled with a novel simulated annealing procedure that allows efficient exploration of multimodal distributions.

We illustrate the merits of our approach in an expression QTL study of monocytes after immune stimulation.

Software is available at https:/github.com/hruffieux/atlasqtl.

\section{Strength of Polygenic Risk Score for Type 2 Diabetes in Arab Population}

\section{Saad $M$}

Qatar Computing Research Institute, Doha, Qatar

Correspondence to: msaad@hbku.edu.qa

Polygenic risk scores (PRS) are most applicable when used for diseases that are preventable or mitigated by early diagnosis. Coronary Artery Disease and Type 2 Diabetes (T2D) are common examples PRS is often applied to.

The applicability of PRS across populations remains challenging. A recent study showed that PRSs developed in one population often do not translate well to other populations. Although interest in studying complex diseases in non-European datasets has increased, Middle Eastern populations are still the least studied.

The aim of this study is to evaluate PRS developed in European populations on an Arab T2D GWAS dataset ( $\sim 500$ cases, $\sim 1200$ controls). Type 2 Diabetes prevalence is $\sim 20 \%$ in the Gulf countries, where consanguinity plays a major role in high prevalence.

Unlike other studies from the region, our study identified known genes (e.g., TCF7L2 and GLIS3). We applied two PRSs derived from Mahajan et al, 2018 (403 SNPs) and Khera et al, 2018 ( 7M SNPs) on our imputed data. Mahajan's PRS had slightly better performance. AUC and p-value of the model “T2D PRS" were 0.636 and 2.07e-21. Adding BMI, age, and sex increases the AUC to 0.806 . Grouping by decile, individuals with PRS values in the highest decile have a 7.43 -fold greater probability to have T2D compared the lowest decile. Our results suggest that PRS generalizes sometimes, here with a higher AUC than in European datasets (0.72, Khera et al).

\section{Two-Stage GWAS Approach for Detecting the Genetic Variants and Their Epistatic Interactions Responsible for Cardiac Aging in Drosophila}

\author{
$\underline{\text { Saha } S^{1}}$, Brun $C^{2}$, Perrin $L^{2}$, Spinelli $L^{2}$ \\ ${ }^{1}$ Laboratoire TAGC/INSERM U1090, Marseille, France; \\ ${ }^{2}$ Laboratoire TAGC/INSERM, Marseille, France \\ Correspondence to: saswati.saha@inserm.fr
}

Senescence is a major determinant of life expectancy in an aging population. As the risk of heart disease is increasing with age, it is becoming critically important to understand the genetic mechanisms of cardiac aging. Genome-wide association studies (GWAS) provide powerful means to identify associations between genetic variants and phenotypes. However, GWAS techniques for detecting epistasis, the interactions between genetic variants associated with phenotypes, are still limited to binary epistatic interactions and most of the existing GWAS approaches cannot handle well the complicated high order interactions.

In this regard, we propose a novel two-stage approach combining a linear mixed-effects model (LMM) and a mixed-effect conditional random forest (MECRF) model to identify the genetic variants responsible for cardiac aging. In the first stage, to alleviate the computational burden, LMM using single marker GWAS 
filters the single nucleotide polymorphism (SNP) markers that have a significant effect on cardiac aging. Then in the second stage, a random forest (RF) model (MECRF) is applied to the SNPs filtered, and the tree structure in RF is utilized to screen the significant high-order SNP interactions attributing to the phenotypic variability. The above method coupled with some biological insights will help us in revealing the gene/protein network(s) of interactions and regulations underlying cardiac aging. It will also help us in understanding the cellular and molecular pathways involved in complex age-related cardiovascular disorders.

Though the above method is applied here for cardiac aging in Drosophila, it can be extended to other pathological diseases in other model organisms. Furthermore, the MECRF approach proposed here is new in GWAS and can have a wide range of applications.

\section{Testing and Correcting for Weak and Pleiotropic Instruments in Two-Sample Multivariable Mendelian Randomisation}

Sanderson $E^{1}$, Spiller $W^{1}$, Bowden $\mathcal{J}^{2}$

${ }^{1}$ University of Bristol, Bristol, UK; ${ }^{2}$ University of Exeter, Exeter, UK

Correspondence to: eleanor.sanderson@bristol.ac.uk

Multivariable Mendelian Randomisation (MVMR) is a form of instrumental variable analysis which estimates the direct effect of multiple exposures on an outcome using genetic variants as instruments. Mendelian Randomisation (MR) and MVMR are frequently conducted using two-sample summary data where the association of the genetic variants with the exposure and outcome are obtained from separate samples. If the genetic variants are only weakly associated with the exposures either individually or conditionally, given the other exposures in the model, then standard inverse variance weighting will yield biased estimates for the effect of each exposure, i.e. there will be 'weak instrument bias'. We develop a two-sample conditional F-statistic to test whether the genetic variants strongly predict each exposure conditional on the other exposures included in a MVMR model. We show that this test is equivalent to the individual level data conditional F-statistic, indicating that the conventional rule-of-thumb of $\mathrm{F}>10$ can be used to test for weak instruments. We then demonstrate how reliable estimates of the causal effect of each exposure on the outcome can be obtained in the presence of weak instruments and pleiotropy, through minimisation of an appropriate heterogeneity Q-statistic. Furthermore, this same Q-statistic yields an exact test for heterogeneity due to pleiotropy. We illustrate our methods and how both the Q and F statistics can be used to guide the choice of genetic instruments and exposure variables in an MVMR analysis with an application to estimate the effect of blood lipid fractions on age related macular degeneration.

\section{Profile-Likelihood Bayesian Model Averaging for Two-Sample Summary Data Mendelian Randomization in the Presence of Horizontal Pleiotropy \\ Shapland $C^{1}$, Zhao $Q^{2}$, Bowden $\mathrm{J}^{3}$ \\ ${ }^{1}$ University of Bristol, Bristol, UK; ${ }^{2}$ University of Cambridge, Cambridge, UK; ${ }^{3}$ University of Exeter, Exeter, UK \\ Correspondence to: chinyang.shapland@bristol.ac.uk}

Two-sample summary data Mendelian randomisation is a popular method for assessing causality in epidemiology, by using multiple genetic variants as instrumental variables. If genetic variants exert pleiotropic effects on the outcome not through the exposure of interest, this leads to heterogeneous and potentially biased estimates of causal effect. It is possible in theory to detect and remove outlying variants, but this can lead to an under-estimation of the standard error and conflate weak instrument bias with pleiotropy.

Rather than detecting and removing outlying estimates or attempting to search all possible subsets, we investigate the use of Bayesian model averaging to preferentially search the space of models with the highest posterior likelihood. We develop a bespoke Metropolis-Hasting algorithm to perform the search and use the profile likelihood of Zhao et al to define a posterior distribution that efficiently accounts for pleiotropic and weak instrument bias. In keeping with the Bayesian framework, our method also allows prior knowledge on the validity of each variant to be seamlessly included. We demonstrate how our general modelling approach can be extended from a standard one-parameter causal model to a two-parameter model, which allows a large proportion of SNPs to violate the Instrument Strength Independent of Direct Effect (InSIDE) assumption.

We use Monte Carlo simulations and real data examples to illustrate our approach and compare it to several related approaches, highlighting its relative strengths and weaknesses in outlier detection and causal estimation.

\section{High-Definition Likelihood Inference of Genetic Correlations across Human Complex Traits}

\author{
Ning $Z^{1}$, Pawitan $Y^{1}$, Shen $X^{2}$ \\ ${ }^{1}$ Karolinska Institutet, Stockholm, Sweden; ${ }^{2}$ University of \\ Edinburgh, Edinburgh, UK \\ Correspondence to: xia.shen@ed.ac.uk
}

Genetic correlation is a central parameter for understanding the shared genetic architecture between complex traits and diseases. Making use of summary-level genome-wide association study (GWAS) data resources, LD Score regression (LDSC) was developed for unbiased estimation of genetic correlation. Though easy to use, LDSC only uses a small part of all the linkage disequilibrium (LD) information in the modeling of summary association statistics. In contrast, by fully accounting for LD information across the human genome, we develop a High-Definition Likelihood (HDL) method to improve the precision in genetic correlation 
estimation. Compared to LDSC, HDL reduces the variance of a genetic correlation estimate by about $60 \%$, which is equivalent to a 2.5-fold increase in sample size. We implement HDL and LDSC to estimate 435 genetic correlations amongst 30 behavioral and disease-related phenotypes measured in UK Biobank. In addition to 154 genetic correlations significant for both methods, HDL identifies another 57 significant genetic correlations compared to only another 2 by LDSC. In summary, HDL brings more power to genome-wide analyses and can better reveal the underlying connections across human complex traits.

\section{Predicting Rare Allele Carriers from Genotyping-Array Data Using Whole Genome Sequencing Data in the Estonian Population}

\author{
Sikka $T^{2}$, Palover $M^{2}$, Nikopensius $T^{2}$, Alver $M^{2}$, Metspalu $A^{2}$, \\ Tõnisson $N^{1}$, Esko $T^{2}$ \\ ${ }^{1}$ University of Tartu, Tartu, Estonia; ${ }^{2}$ University of Tartu, \\ Tartu, Estonia \\ Correspondence to: timo.tonis.sikka@ut.ee
}

Introduction: Genetic imputation works well with frequent alleles (minor allele frequency $>1 \%$ ), however, its predictive accuracy drops for rare genetic variants which can also play a role for eventual development of diseases. Exceptions to such limitations are endogamous populations like Estonia. Currently, 50,000 participants of Estonian biobank have been genotyped, with a total of 200,000 participants genotyped by June 2020, and their genetic profiles will be added to electronic health records. We performed a study to predict and verify rare mutation carriers for severe disease predispositions such as familial breast cancer (in genes BRCA1 and $B R C A 2)$ and familial hypercholesterolemia $(A P O B)$ using long range haplotyping (LRH) and "surrogate parent" theory.

Materials and methods: We used whole genome sequence data of 2,244 and genotyped data of 15,416 participants of the Estonian biobank. Whole genome sequencing (WGS) with 30x coverage was carried out at Broad Institute using Illumina HiSeq xTen platform.

Results: WGS identified 14, 4 and 6 mutations carriers for $B R C A 1, B R C A 2$ and $A P O B$, respectively. We identified 16 mutations carriers for $B R C A 1$ and 3 for $B R C A 2$, and 5 carriers for $A P O B$ using LRH among genotyped samples. We failed to find carriers for additional 6 out of 9 mutations, highlighting these as recent mutations and only present in limited historical lineages.

Conclusion: LRH is a cost-effective approach to predict additional rare mutation carriers for different disease predispositions from genotyped data in endogamous populations and will be important in the process of reporting clinically relevant mutations to Estonian biobank participants.

\section{Integrating Gene Expression and Metabolome Data Identifies Genes Causally Acting on Metabolite Concentrations}

Sonmez Flitman $R$, Khalili B, Rueedi R, Bergmann S

University of Lausanne, Lausanne, Switzerland

Correspondence to: reyhan.sonmez@unil.ch

We performed an untargeted metabolome-wide and transcriptome-wide association analysis to identify genes potentially influencing the human metabolome. We characterized the urine metabolome of 555 Caucasian individuals using $1 \mathrm{H}$ nuclear magnetic resonance spectroscopy (NMR) and used RNAseq to quantify gene expression profiles of lymphoblastoid cell lines derived from the same set of subjects. We took an untargeted approach allowing for data-driven discovery by using binned NMR spectra. We identified a number of significant associations between NMR spectral features and genes using pairwise linear regression. Next, we used metabomatching to identify most likely metabolites underlying these novel associations. This approach revealed two genes, ALMS1 and HPS1, that are linked to two metabolites $\mathrm{N}$-Acetyl L-Aspartate and trimethylamine respectively. Indeed, a number of metabolome-wide genome-wide association studies (mGWAS) had already suggested the locus of ALMS1 to be involved in regulation of $\mathrm{N}$-acetylated compounds, yet were not able to identify unambiguously the exact metabolite, nor to disambiguate between ALMS1 and NAT8, another gene found in the same locus, as the mediator gene. The HPS1 locus had also previously been implicated by $\mathrm{mGWAS}$ to modulate trimethylamine yet $\mathrm{mG}$ WAS could not disambiguate between HPS1 and PYROXD2, another gene found in the same locus. Finally, we investigated ALMS1 and HPS1 genes' causal effect on the respective metabolite concentrations by using Mendelian randomization. Our study provides evidence that the integration of metabolomics with gene expression data can support mGWAS analysis, helping to identify the most likely gene involved in the modulation of the metabolite concentration.

\section{Heterogeneity in Obesity and Its Consequences on Health \\ Sulc J', Sonrel $A^{2}$, Kutalik $Z^{1}$ \\ ${ }^{1}$ University of Lausanne, Lausanne, Switzerland; ${ }^{2}$ University of Zürich, Zurich, Switzerland \\ Correspondence to: jonathan.sulc@unil.ch}

Obesity-associated SNPs have mostly been tested for only one trait in isolation and their joint impact on fat/lean mass accumulation/distribution and downstream effects on health and quality of life remain poorly understood.

We applied principal component analysis on the effect estimates of SNPs on fourteen measures of body morphology from the UK Biobank to identify the genetic axes of variation giving rise to differences in body shape and composition. This provided three independent components affecting overall body size, body 
composition, and body fat distribution, respectively. Our method developed for composite trait Mendelian randomization revealed that these components have both shared and specific effects on health outcomes and quality of life. Of particular interest is the component shifting subcutaneous to visceral fat, which was increased the risk of many obesity-related diseases (such as diabetes, hypertension, hypercholesterolemia, and coronary artery disease) despite being neutral in terms of body mass index and total body fat percentage. A shift in mass from lean to adipose prominently impacted lifestyle, increasing alcohol consumption and smoking. Sex-stratified analyses revealed that increased body size leads to hypothyroidism and decreased socio-economic status in women alone. Enrichment analyses suggest that brain and nervous tissues contribute most to body size and composition, whereas genes highly expressed in adipose tissue and during development are more likely to affect body fat distribution.

These genetic components provide a basis to better understand the mechanisms underlying inter-individual differences in body fat accumulation and distribution, as well as the consequences they have on health.

\section{Reducing the GWAS Inflation for Structured Samples Using Linkage Disequilibrium Score Regression}

Svishcheva $G^{2}$, Tsepilov $Y^{1}$

${ }^{1}$ Institute of Cytology and Genetics, SB RAN, Novosibirsk, Russia;

${ }^{2}$ Institute of Cytology and Genetics, SB RAS, Novosibirsk, Russia

Correspondence to: gulsvi@mail.ru

The recent technological improvements in genome-wide SNP genotyping have contributed to the rapid accumulation of GWAS data (with the high density of SNP coverage and big sample size). To analyze structured samples, GWAS methods usually use a polygenic mixed model (PMM) under the null hypothesis of no association. However, these methods are not able to adequately analyze big GWAS data due to the lack of effective models including factors that appear only when analyzing big data. As a result, many large GWASs using a PMM show a trend that the distribution of summary z-score statistics is inflated compared to the theoretical standard distribution under the null hypothesis. Linkage disequilibrium (LD) score regression can explain the GWAS inflation as the result of confounding due to inadequate control for population stratification, cryptic relatedness or unaccounted polygenic background.

Here we propose a new statistical inflation-reducing approach that corrects GWAS z-score statistics obtained taking into account the population structure of the sample. This approach is based on LD score regression under the assumption that GWAS z-score statistics are obtained using a PMM. The basic idea of LD score regression is that inflation in the $\mathrm{z}$-score statistics usually tends to be higher in regions with high $\mathrm{LD}$, and inflation due to unaccounted population stratification is uncorrelated with LD. This can be used to separate inflation due to confounding from inflation due to polygenicity.

This work was supported by the RFBR Grant (20-04-00464).

48th European Mathematical Genetics Meeting

\section{A New Method for Combining of Genetically Correlated Traits by Maximizing of Their Shared Heritability}

\author{
Svishcheva $G^{1}$, Tiys $E^{2}$, Feoktisova $S^{2}$, Elgaeva $E^{2}$, \\ Sharapov $S^{2}$, Tsepilov $Y^{2}$ \\ ${ }^{1}$ Institute of Cytology and Genetics, SB RAS, Novosibirsk, Russia; \\ ${ }^{2}$ Institute of Cytology and Genetics, SB RAN, Novosibirsk, Russia \\ Correspondence to: gulsvi@mail.ru
}

In the era of large sample sizes available for genome wide association studies (GWAS), it becomes clear that a remarkable number of human traits/diseases are highly genetically correlated. In other words, many groups of traits have a significant level of shared genetic background. There is a big temptation to be able to study this background by analyzing a new aggregate trait that shows the shared heritability of the studied traits. We propose a new multi-step strategy to the SNP-based association analysis of multiple genetically correlated traits. This approach focuses on a weighted linear combination of the traits by maximizing of heritability explained by SNPs associated with all the traits (named as 'shared' SNPs). The first step is to decompose a matrix of genetic correlations between traits into two matrices so that one of them is explained by shared SNPs, while the other represents the genetic correlations formed by the unique genetic background of each trait. The second step allows estimating the weights of traits by maximizing their shared heritability explained only the shared SNPs. Finally, in the last step, GWAS of shared heritability is performed for a weighted linear combination of single-trait GWAS summary statistics. We assessed this approach using both simulations and real data and showed that in most of the cases, it has a bigger analysis power compared to other methods and allows extracting the shared heredity with acceptable precision.

This work was partially supported by the RFBR grant (20-04-00464).

\section{Causal Relationships between Dietary Items and Blood Metabolites Using Mendelian Randomization}

\author{
Taba $N^{2}$, Fischer $K^{2}$, Esko $T^{2}$, Metspalu $A^{2}$, Pirastu $N^{1}$ \\ ${ }^{1}$ University of Edinburgh, Edinburgh, UK; ${ }^{2}$ University of Tartu, \\ Tartu, Estonia \\ Correspondence to: nele.taba@ut.ee
}

Nutrition plays an important role in the development and progress of several diseases, which in turn creates high burden for individuals, society and health-care. In many cases the mechanism by which food acts on health is still unclear. One of the candidates for filling this gap are blood metabolites. Thus detecting causal relationships between dietary choices and biomarkers might give more insight into the mechanism by which food affects health.

To try to answer these questions we turned to Mendelian Randomization. The food exposure SNPs were selected amongst those coming from a recent food consumption GWAS performed in UK biobank (Pirastu et al., 2019). We included 25 individual and 14 
principal component (PC) traits reflecting different levels of dietary patterns. To identify which SNPs directly influence the exposure of interest, we used additionally the Corrected to Raw ratio filtering (Pirastu et al., 2019). As outcomes we used the results from a GWAS conducted on 123 blood metabolites (Kettunen et al., 2016).

After correction for multiple testing, 48 food-metabolite pairs were significant. For example, Psychoactive PC1 (reflects higher consumption of both alcohol and coffee) increases cholesterol levels in low density lipoprotein and in intermediate density lipoprotein, which are both known to have adverse effects on cardiovascular health. Furthermore, we saw a significant effect of Psychoactive PC1 on Apolipoprotein-B, suggesting that part of the effect of alcohol on CVD may be mediated through this mechanism. This is indicating the potential of our approach in explaining food-health relationships.

\section{Multivariate Genomic Scan of Human Ageing Traits Reveals Novel Loci and Identifies Haem Metabolism as a Human Ageing Pathway}

\author{
Timmers $P^{2}$, Wilson $J^{2}$, Joshi $P^{2}$, Deelen $J^{1}$ \\ ${ }^{1}$ Max Planck Institute for Biology of Ageing, Cologne, Germany; \\ 2University of Edinburgh, Edinburgh, UK \\ Correspondence to: paul.timmers@ed.ac.uk
}

Ageing phenotypes, such as years lived in good health (healthspan), total years lived (lifespan), and survival until an exceptional old age (longevity), are of interest to us all but require exceptionally large sample sizes to study genetically. By combining existing genome-wide association summary statistics for healthspan, lifespan, and longevity in a multivariate framework, we increased statistical power and showed that the traits share more than $50 \%$ of their underlying genetics. Our method identified 10 genomic loci which influence all three phenotypes, of which five (near FOXO3, SLC4A7, LINC01259, ZW10, and FGD6) are reported for the first time at genome-wide significance. The majority of these loci are associated with cardiovascular disease and several show signs of antagonistic pleiotropy. Using gene expression data, we implicated the expression of 59 genes and found this set of genes to be enriched for ageing pathways previously highlighted in model organisms, such as the response to DNA damage, apoptosis, and homeostasis. Finally, we identify a new pathway worthy of further study: haem metabolism.

\section{The Genetics of Retinal Microvascular Features}

\section{Tomasoni M Hersch M, Bergmann S}

University of Lausanne, Lausanne, Switzerland

Correspondence to: mattia.tomasoni@unil.ch

The microvasculature present on the retina of the human eye can inform us about disease. Features such vessel caliber and tortuosity are known to reflect systemic and cardiovascular health. I use Machine Learning on a space defined by retinal microvascular features to predict blood pressure and other health-related risk factors. I leverage the UKBiobank dataset of retinal images and genotypes (comprising around 180k individuals) to investigate whether these features have a genetic component.

\section{Loci and Genes Involved in Chronic Musculoskeletal Pain Identified via Analysis of Genetically Orthogonal Pain Phenotypes}

\author{
Tsepilov $Y^{3}$, Freidin $M^{1}$, Shadrina $A^{3}$, Elgaeva $E^{3}$, Sharapov $S^{3}$, \\ van Zunder $\mathcal{J}^{2}$, Karssen $L^{4}$, Suri $P^{5}$, Williams $F^{1}$, Aulchenko $Y^{3}$ \\ ${ }^{1}$ King's College London, London UK; ${ }^{2}$ Maastricht University \\ Medical Centre, Maastricht, The Netherlands; ${ }^{3}$ Novosibirsk State \\ University, Novosibirsk, Russia; ${ }^{4}$ PolyOmica, 's-Hertogenbosch, \\ Netherlands; ${ }^{5}$ University of Washington, Seattle, WA, USA \\ Correspondence to: drosophila.simulans@gmail.com
}

Chronic musculoskeletal pain has a negative impact on all aspects of human life. Genetic studies of pain are complicated by the high complexity and heterogeneity of pain phenotypes. In this research, we aimed to reduce phenotype heterogeneity and reveal genes and pathways shared by chronic musculoskeletal pain at four locations: back, neck/shoulder, hip, and knee. Our study was based on the results of genome-wide association studies performed using UKBiobank data with a total sample size of 456,000 individuals. We applied principal component analysis based on the matrix of genetic covariances between the studied pain traits and constructed four genetically independent phenotypes (GIPs). The leading GIP (GIP1) explained the largest proportion of the genetic variance $(78.4 \%)$. We identified and replicated five loci associated with GIP1; and one locus associated with GIP2. The genes confidently prioritized for the GIP1-associated loci were SLC39A8, ECM1, and FOXP2. For the remaining two GIP1-associated loci, we proposed several candidates but were unable to prioritize any of them convincingly. The most likely causal gene in the locus associated with GIP2 was GDF5. For GIP1, gene set/tissue/cell type enrichment analyses identified multiple terms related to the nervous system. Genetic correlation analysis revealed a genetic overlap between GIP1 and osteoarthritis as well as a set of anthropometric, sociodemographic and psychiatric/personality traits. We suggest that GIP1 represents a biopsychological component of chronic musculoskeletal pain, related to physiological and psychological aspects and possibly reflecting pain perception and processing. The research has been conducted using the UKBiobank (project \#18219). 


\section{Relationship with Breast Cancer Subtypes and Potential Predictive Factors from a North Cyprus Cohort Study}

Ulgen A

Girne American University, Karmi, Cyprus

Correspondence to: ayseulgen1@gmail.com

More than 300 breast cancer patients with subtype information are surveyed from the State Hospital in Nicosia between 2006-2015 for their demographic, reproductive, genetic, epidemiological factors, which represented $40 \%$ of total breast cancer cases in the archives during this period. The breast cancer subtypes, Estrogen receptor $(\mathrm{ER})+/$-, Progesterone receptor $(\mathrm{PR})+/$-, and human epidermal growth factor 2 (HER2) +/- status, are determined. Single and multiple variable, regularized regressions, LASSO, with predictive factors as independent variables, breast cancer subtypes as dependent variables are conducted. Despite the fact that our cohort differs significantly from larger cohorts such as the Breast Cancer Family Registry, in age, menopause status, age of menarche, parity, education level, oral contraceptive use, breast feeding, the distribution of breast subtypes is not significantly different. The subtype distribution in our cohort is also not different from another study on a Turkish cohort. Using regularized regressions, we show that the ER+ subtype is positively related to post-menopause and negatively associated with hormone therapy; ER+/PR+ is positively associated with breast feeding, and negatively associated with hormone therapy status. HER2+, which itself is negatively correlated with $\mathrm{ER}+$ and $\mathrm{ER}+/ \mathrm{PR}+$, is positively related to having first-degree-relative with cancer, and negatively associated with post-menopause. Regressions identify older age to be positively correlated to $\mathrm{ER}+$ and $\mathrm{ER}+/ \mathrm{PR}+$, negatively correlated to HER2+. To conclude, assuming ER+ and ER+/PR+ to have better prognostic, post-menopause and breast-feeding are beneficial, hormone therapy treatment is detrimental.

\section{Large Scale Livestock Genomic Data Can Be Used to Accurately Detect Recombination in Hundreds of Thousands of Individuals. \\ Whalen $A^{2}$, Johnsson $M^{2}$, Ros-Freixedes $R^{2}$, Chen $C-Y^{1}$, Herring $W^{1}$, Hickey $\mathcal{J}^{2}$ \\ ${ }^{1}$ Genus PLC, Basingstoke, UK; ${ }^{2}$ The Roslin Institute, University of Edinburgh, Edinburgh, UK \\ Correspondence to: awhalen@roslin.ed.ac.uk}

Estimating the frequency and position of recombination is important for understanding the evolutionary history of the genome and detecting adaptive loci. Livestock pedigrees, particularly those with large numbers of genotyped individuals, present a valuable resource for understanding the recombination landscape. However, scaling statistical methods to detect recombination, such as multi-locus iterative peeling, in pedigrees of these sizes is challenging.
In this project, we developed a fast and accurate approximation of multi-locus iterative peeling which can scale to pedigrees with hundreds of thousands of genotyped individuals. We then applied this method to estimate where recombination occurred in 150,000 pigs across 9 commercial breeding lines.

We found that when using this data, we could accurately estimate (i) the total genetic map length for each chromosome, (ii) the within-chromosome recombination landscape, and (iii) sexdifferences in both the genetic map length and recombination landscape. We found large similarities between the number and locations of recombination between breeding lines. Recombination was mildly heritable $(\mathrm{h} 2<0.1)$, and a GWAS found three regions that were associated with an individual's genome-wide recombination rate. This project represents the feasibility of using largescale pedigree and genomic data to accurately estimate recombination, and provides an important advance in applying multi-locus iterative peeling to populations of hundreds of thousands of genotyped individuals.

\section{Designing a Tanzanian Specific SNP Array Add-On to Capture Population-Specific Genetic Variations and to Improve Genotype Imputation}

$\underline{X u Z^{1}}$, Rüeger $S^{1}$, Portevin $D^{2}$, Gagneux $S^{2}$, Fellay $J^{1}$

${ }^{1}$ School of Life Sciences, École Polytechnique Fédérale de Lausanne, Lausanne, Switzerland; ${ }^{2}$ Swiss Tropical and Public

Health Institute, Basel, Switzerland

Correspondence to: zhi.xu@epfl.ch

African ancestry is often underrepresented in large-scale genomic studies, and the genetic diversity of various local populations has yet to be captured. To better capture the genetic variability of the Tanzanian population, we have developed a pipeline for the selection of single nucleotide polymorphisms (SNPs) to be added to the H3Africa genotyping array to maximize coverage of common variation.

We obtained whole genome sequencing (WGS) data of 118 individuals from various tribes within Tanzania as part of the TBDAR cohort and split them into a training and a testing set. To measure baseline imputation performance and identify poorly imputed SNPs, we masked our testing set to include only SNPs captured by the H3Africa array, and imputed using the African Genome Resources (AFGR) reference panel and a reference panel constructed from our training set of WGS samples. Within our training set, we applied a Mutual Information (MI) maximizationbased algorithm to select optimal tag SNPs for poorly imputed SNPs, incorporating MI of existing tags, probe quality scores, and the required number of probes. After the addition of 4659 SNPs, weobserved anincreaseinmeanimputation quality $(\triangle \mathrm{INFO}=0.063)$ for SNPs with MAF $>0.05$. We also observed an increase in mean correlation between imputed SNP dosages and the ground truth WGS dosages $\left(\Delta r^{2}=0.034\right)$.

Our approach accurately selected add-on SNPs which captured population-specific genetic variations and contributed to improved imputation performance, thus likely to increase the power of genome-wide association studies in the Tanzanian population. 


\section{Triangulation of Analysis Strategies Links Complex Traits to Specific Tissues and Cell Types}

\author{
Yang $Z^{2}$, Xu $W^{2}$, Zhai $R^{2}, L i T^{2}$, Ning $Z^{1}$, Pawitan $Y^{1}$, Shen $X^{3}$ \\ ${ }^{1}$ Karolinska Institutet, Stockholm, Sweden; ${ }^{2}$ Sun Yat-sen \\ University, Guangzhou, China; ${ }^{3}$ University of Edinburgh, \\ Edinburgh, UK \\ Correspondence to: yangzhj28@mail2.sysu.edu.cn
}

Linking complex traits and diseases to their relevant tissues and cell types of humans is of great importance, providing useful etiological and functional insights for understanding the regulatory mechanisms of the traits and diseases. In recent years, different methods have been developed to detect such links by integrating GWAS results and gene expression data of tissues and cells. However, distinct results from these methods generate confusion while no gold standard is currently accepted, making it difficult to evaluate the discoveries. Here, we estimate the operating characteristics of four methods via maximum likelihood in the absence of a gold standard and scored the discoveries using the estimated specificities to provide more specific associations. By integrating GWAS summary statistics with gene expression data and eQTL data from the Genotype-Tissue Expression (GTEx) project, we establish tissue-trait associations with higher specificity, including obvious positive controls such as the connections between human height and skeletal muscle, blood lipids and liver, and psychiatric disorders and brain tissues. Furthermore, three of the four methods are able to take advantage of single-cell RNA sequencing data so that we can establish more specific associations between complex traits and cell types. Our results provide higher confidence in comprehending the underlying tissues and cell types for complex traits, assisting future experimental designs and clinical research.

\section{Extracellular Vesicles with Specific Surface Proteins Are Associated with Waist Visceral Fat}

\author{
Shen $X^{3}$, Zhai $R^{2}$, Yang $Z^{2}$, Li $T^{2}$, Ning $Z^{1}$, Pawitan $Y^{1}$, \\ Wilson $\mathcal{J}^{3}$, Wu $D^{4}$ \\ ${ }^{1}$ Karolinska Institutet, Stockholm, Sweden; ${ }^{2}$ Sun Yat-sen \\ University, Guangzhou, China; ${ }^{3}$ University of Edinburgh, \\ Edinburgh, UK; ${ }^{4}$ Vesicode AB, Solna, Sweden \\ Correspondence to: zhairr@mail2.sysu.edu.cn
}

The field of genomics has spent tremendous efforts trying to discover the genetic regulation and mechanisms underlying complex traits and diseases. While many associations have been found, our understanding remains far from satisfactory, due to the high complexity of genetic architecture. Omics techniques provide an opportunity to look at the problem with better resolution, e.g. integration of genome-wide association results and single-cell omics information can sometimes give us a clue in which cells our diseases may develop.

As a proxy of cell-level biology, extracellular vesicles (EV) have become popular candidate biological complexes to study the source of cell regulation of complex diseases. EVs carry a lot of biological information and are largely enriched in human plasma. Here, we utilized a novel technology to detect the presence of 120 candidate proteins across millions of single EVs. By integration with GWAS summary statistics, we identified combinations of coding genes for the EV surface proteins being associated with obesity-related traits such as waist circumference. We subsequently verified such associations by quantifying these EVs with the particular protein profiles and testing their associations with body fat measured by DEXA scans in 96 individuals from Orkney. We found that the lower abundance of EVs that carry both ITGB6 and ITGB8 indicates larger waist circumference, as well as more waist visceral fat. Our findings provide the first evidence that EVs with specific surface proteins are associated with obesity, suggesting visceral fat can be tested using plasma and shedding light on future EV biomarker discovery.

\section{Physical Activity Reduces Colorectal Cancer Risk Independent of BMI: A Mendelian Randomisation Study}

Zhang $X^{1}$, Theodoratou $E^{1}, L i X^{1}, M$ Farrington $S^{4}$, J Law $P^{3}$, Broderick $P^{3}$, Walker $M^{2}, M B$ Rees $J^{5}$, S Houlston $R^{3}$, PM Tomlinson $1^{6}$, Campbell $H^{1}, G$ Dunlop $M^{2}$, Timofeeva $M^{2,7}$

${ }^{1}$ Centre for Global Health Research, Usher Institute of Population Health Sciences and Informatics, The University of Edinburgh, UK; ${ }^{2}$ Colon Cancer Genetics Group, Medical Research Council Human Genetics Unit, Institute of Genetics and Molecular Medicine, Western General Hospital, University of Edinburgh, UK; ${ }^{3}$ Division of Genetics and Epidemiology, The Institute of Cancer Research, London, UK; ${ }^{4}$ Colorectal Cancer Genetics Group, CRUK Edinburgh Centre, Institute of Genetics and Molecular Medicine, Western General Hospital, University of Edinburgh, UK; ${ }^{5}$ Edinburgh Clinical Trials Unit, Centre for Global Health Research, Usher Institute of Population Health Sciences and Informatics, The University of Edinburgh, UK; ${ }^{6}$ Cancer Genetics and Evolution Laboratory, Institute of Cancer and Genomic Sciences, University of Birmingham, Vincent Drive, Edgbaston, Birmingham, UK; ${ }^{7}$ DIAS, Danish Institute for Advanced Study, Department of Public Health, University of Southern Denmark, Odense, Denmark

X.Z. and E.T. contributed equally to this study.

Correspondence to Maria.Timofeeva@igmm.ed.ac.uk or malcolm.dunlop@ed.ac.uk

Evidence from observational studies suggests a protective role for physical activity (PA) against colorectal cancer (CRC) risk. However, evidence from randomised clinical trials is lacking and the role of body mass index (BMI) is not clear. We conducted a Mendelian randomisation (MR) study to examine whether the observed association between PA and CRC risk is causal and independent of BMI.

Common genetic variants associated with self-reported, accelerometer measured PA and BMI were used as instrumental variables (IVs) in this MR study. We analysed the effect of the IVs in a large CRC GWAS that included 31197 cases and 61770 controls. 
The inverse variance weighted (IVW) method was applied as the main analysis method.

Our results demonstrated a protective effect between self-reported MVPA and CRC risk per one standard deviation (SD) increase of self-reported MVPA independent of BMI (OR [95\% CI]: $0.52[0.29,0.93])$. The effect between accelerometer measured PA and CRC risk was also supportive of inverse association per one SD increase of accelerometer measured PA independent of BMI (OR [95\% CI]: $0.60[0.41,0.88]$ ). The proportion of effect mediated through BMI were $2.2 \%$ (95\%CI: $2.0 \%, 2.4 \%)$ and $9.1 \%$ (95\%CI: 6.7\%, 11.4\%) for MVPA and accelerometer measured PA respectively.

The findings of this large MR study quantified the total causal effect of both subjectively and objectively measured physical activity on CRC risk and the proportion of effect mediated through $\mathrm{BMI}$, which showed that PA can reduce CRC risk independent of BMI. Therefore, at a population level, these findings provide strong reinforcing evidence to support public health policy measures that encourage exercise.

\section{Mapuche Ancestry and Gallbladder Cancer Risk: Causality or Endogeneity Bias?}

\section{Zollner L}

Institute of Medical Biometry and Informatics, University of Heidelberg, Heidelberg, Germany

Correspondence to: zollner@imbi.uni-heidelberg.de

The incidence of gallbladder cancer (GBC) in Chile is the highest in the world and has been associated with the individual proportion of Native American ancestry, in particular with Mapuche ancestry-Mapuche Chileans live in the south of the country. Since association does not imply causation, individuals with large proportions of Mapuche ancestry could show specific risk exposures and worse access to the health system. Therefore, we took advantage of ancestry informative markers (AIMs) and applied Mendelian randomisation (MR) to test causality between Mapuche ancestry and GBC risk.

By estimating the informativeness for assignment measure (IN), we selected IN-AIMs with distinct allele frequencies in Mapuche and other original Chilean populations, namely Europeans, Africans and Native Americans from northern Chile (Aymara and Quechua). The selected IN-AIMs were utilized as instrumental variables for the individual proportion of Mapuche ancestry in two-sample-MR (sample 1: 1,800 Chileans from the whole country, sample 2: 255 Chilean case-control pairs). We found evidence for a causal effect of Mapuche ancestry on GBC risk: inversevariance-weighted OR per $1 \%$ increase in Mapuche proportion $1.02,95 \%$ CI (1.01-1.03), Pval $=0.0001$. Radial-MR was applied to identify and subsequently exclude outlying instruments and we checked different combinations of genetic principal components to examine the potential effects of population stratification unrelated to Mapuche ancestry. The results of these sensitivity analyses confirmed the causal association between Mapuche ancestry and GBC risk. We are currently applying two-step-MR to investigate the mediating effect of BMI on this causal relationship. 
Adesoji, O. 204

Ala-Korpela, M. 220

Alasoo, K. 204

Allen, R. 214, 215

Almorza, D. 205

Alver, M. 224

Anasanti, M. 212

Aryaman, J. 211

Asimit, J. 205

Aulchenko, Y. 226

Bacq-Daian, D. 207

Baldauf, H. 206

Balkhiyarova, Z. 220

Barysenka, A. 220

Bergmann, S. 224, 226

Berrandou, T. 205

Beuchel, C. 206

Blanché, H. 207

Bocher, O. 206

Böhringer, S. 206

Borges, M. 207

Bouatia-Naji, N. 205

Bowden, J. 223

Branicki, W. 212

Braybrooke, R. 215

Broderick, P. 228

Brun, C. 222

Burch, K. 207

Butterworth, A. 216

Campbell, H. 228

Ceglarek, U. 206

Chen, C.-Y. 227

Claringbould, A. 220

Clark, D. 211

Cordell, H. 211

Corvin, A. 218

Corvol, J.-C. 208

Dandine-Roulland, C. 207

Darrous, L. 208

Davey Smith, G. 221

Day, F. 219

de Wreede, L. 206

Dedoussis, G. 220

Deelen, J. 226

Delaneau, O. 221

Deleuze, J.-F. 207

Dila, G. 208
Djebali, S. 208

Dong, N. 207

Draisma, H. 209, 220

Dunlop, M.G. 228

Ek, W. 210, 212

Elgaeva, E. 225, 226

Elo, L. 213

Enroth, S. 210, 216

Erdmann, J. 209

Erik Ojavee, S. 218

Eriksson, N. 216

Eriksson, P. 216

Esko, T. 214, 215, 216, 219, 224, 225

Fahy, W. 214

Farrington, S.M. 228

Fellay, J. 227

Feoktisova, S. 225

Fingerlin, T. 215

Fischer, K. 209, 214, 217, 218, 219, 225

Flores, C. 215

Folkersen, L. 216

Franke, L. 216, 220

Freidin, M. 226

Freund, M. 207

Fu, J. 216

Gaffney, A. 213

Gagneux, S. 227

Gaunt, T. 219

Génin, E. 206, 210

Gilly, A. 220

Gola, D. 209

Goudet, J. 210

Grzeszkowiak, E. 219

Guggenheim, J. 219

Guillen-Guio, B. 215

Gustafsson, S. 216

Gyllensten, U. 216

Hansson, O. 216

Haycock, P. 207

Hayward, C. 214, 216

Hernández, N. 205

Heron, E. 218

Herring, W. 227

Hersch, M. 226
Herzig, A. 210

Hickey, J. 227

Hingorani, A. 207

Hofmeister, R. 221

Höglund, J. 210

Hoitzing, H. 211

Holmes, M. 221

Houlston, R.S. 228

Howey, R. 211

Hubbard, R. 214, 215

Hysi, P. 212

Imamura, F. 219

Ingelsson, E. 216

Insalata, F. 211

Järvelin, M.-R. 212, 220

Jenkins, G. 214, 215

Johansson, Å. 210, 212, 216

Johnson, R. 207

Johnsson, M. 227

Jones, N. 211

Joshi, P. 211, 214, 216, 219, 226

Kaakinen, M. 209, 212, 220

Käärik, M. 214

Kalnapenkis, A. 215, 216

Kals, M. 215

Kaminski, N. 214, 215

Kandus, M. 205

Kangombe, A. 214

Karaleftheri, M. 220

Karlsson, T. 210, 212

Karssen, L. 226

Katsara, M.-A. 212

Katsoula, G. 213

Kayser, M. 212

Kelly, C. 213

Kerimov, N. 204

Khalili, B. 224

Khan, S. 213

Kichaev, G. 207

Kirsten, H. 206

Klaric, L. 214

Kolberg, L. 204

Kolde, A. 214

König, I. 209

Kousathanas, A. 218
Kraven, L. 214

Kutalik, Z. 208, 219, 220, 224

Läll, K. 209

Landini, A. 214

Langenberg, C. 216

Lauc, G. 214

Law, P.J. 228

Lawlor, D. 207

Le Floch, E. 207

Le Folgoc, G. 210

Leavy, O. 215

Lepamets, M. 215

Lepik, K. 220

Li, T. 216,228

Li, X. 228

Löffler, M. 206

Ludwig, T. 206

Lysenkov, V. 213

Ma, S.-F. 215

Macdonald-Dunlop, E. 216, 217

Mägi, R. 215

Maher, T. 214,215

Mälarstig, A. 216

Mancuso, N. 207

Mändul, M. 209, 217

Manuel, A. 207

Marenne, G. 206

Martinez, M. 208

May-Wilson, S. 217

Mayer, C. 208

McDonnell, C. 219

McLaughlin, R. 213

Metspalu, A. 224, 225

Meyer, V. 207

Michaelsson, K. 216

Michel, C. 208

Möls, M. 217

Molyneaux, P. 214, 215

Mounier, N. 208, 219

Müller-Myhsok, B. 209

Naret, O. 217

Navaratnam, V. 214

Navarro, P. 214, 221

Newcombe, P. 205

Nikopensius, T. 224 
Ning, Z. 223, 228

Noth, I. 215

Nothnagel, M. 204, 212

Oballa, E. 214

Ojavee, S. 218

Oldham, J. 215

Ong, K. 219

Ormond, C. 218

Palover, M. 224

Pasaniuc, B. 207

Patxot, M. 218

Pawitan, Y. 223, 228

Perdry, H. 219

Perrin, L. 222

Perry, J. 219

Peters, J. 216

Pirastu, N. 217, 219, 225

Plotnikov, D. 219

Poch, O. 208

Porcu, E. 220

Portevin, D. 227

Pospiech, E. 212

Prada Oliveira, A. 205

Prokopenko, I. 209, 212, 220

Pupko, I. 220

Rafati, N. 210

Rask-Andersen, M. 210, 212

Rees, J.M.B. 228

Repetto, L. 221

Resch, L. 207

Reymond, A. 220

Ribeiro, D. 221
Richardson, T. 221

Ripp, R. 208

Robinson, M. 218

Ros-Freixedes, R. 227

Rubinacci, S. 221

Rueedi, R. 224

Rüeger, S. 227

Ruffieux, H. 222

Saad, M. 222

Saha, S. 222

Sahbatou, M. 207

Saini, G. 214

Salerno, J. 205

Sanderson, E. 221, 223

Sandhu, M. 205

Sandron, F. 207

Santoni, F. 220

Sayers, I. 214

Schetelig, J. 206

Schmitz, D. 212

Scholz, M. 206

Schunkert, H. 209

Schwartz, D. 215

Sebert, S. 220

Shadrina, A. 226

Shapland, C. 223

Sharapov, S. 225, 226

Shen, X. 216, 221, 223, 228

Shi, H. 207

Siegbahn, A. 216

Sikka, T. 224

Smith, G. 216

Sonmez Flitman, R. 224

Sonrel, A. 224
Southam, L. 213

Spiller, W. 223

Spinelli, L. 222

Steinberg, J. 213

Sulc, J. 224

Suri, P. 226

Svishcheva, G. 225

Taba, N. 219, 225

Taylor, A. 214

Teunissen, C. 216

Theodoratou, E. 228

Thiery, J. 206

Thompson, J. 208

Tilling, K. 221

Timmer, P. 211

Timmers, P. 216, 226

Timofeeva, M. 228

Tiys, E. 225

Tobin, M. 214

Tomasoni, M. 226

Tomlinson, I.P.M. 228

Tõnisson, N. 224

Tönjes, A. 206

Tournier-Lasserve, E. 206

Trbojevic-Akmacic, I. 214

Trejo Banos, D. 218

Tsafantakis, E. 220

Tsepilov, Y. 225, 226

Ulgen, A. 227

van der Burg, L. 206

van Zunder, J. 226

Velo-Suárez, L. 210
Võsa, U. 215

Vuckovic, F. 214

Wain, L. 214, 215

Walker, M. 228

Wallace, C. 205

Wallentin, L. 216

Walsh, S. 212

Weir, B. 210

Whalen, A. 227

Wheeler, E. 216

Whyte, M. 214

Wielscher, M. 220

Wilkinson, J.M. 213

Williams, C. 219

Williams, F. 226

Wilson, J. 211, 214, 216, 217 , 219, 221, 226, 228

Wu, D. 228

Xu, W. 228

Xu, Z. 227

Yang, Z. 216, 228

Yeo, A. 214

Zeggini, E. 213, 216, 220

Zhai, R. 216, 228

Zhang, X. 228

Zhang, Y. 215

Zhao, Q. 223

Zheng, J. 219

Zhernakova, D. 216

Zollner, L. 229

Zudina, L. 220 